\title{
A review about Invariance Induced Gravity: Gravity and Spin from Local Conformal-Affine Symmetry
}

\author{
S. Capozziello and M. De Laurentis \\ Dipartimento di Scienze Fisiche, Università di Napoli "Federico II" and INFN Sez. di Napoli, \\ Compl. Univ. Monte S. Angelo, Ed.N, Via Cinthia, I-80126 Napoli, Italy
}

\begin{abstract}
In this review paper, we discuss how gravity and spin can be obtained as the realization of the local Conformal-Affine group of symmetry transformations. In particular, we show how gravitation is a gauge theory which can be obtained starting from some local invariance as the Poincaré local symmetry. We review previous results where the inhomogeneous connection coefficients, transforming under the Lorentz group, give rise to gravitational gauge potentials which can be used to define covariant derivatives accommodating minimal couplings of matter, gauge fields (and then spin connections). After we show, in a self-contained approach, how the tetrads and the Lorentz group can be used to induce the spacetime metric and then the Invariance Induced Gravity can be directly obtained both in holonomic and anholonomic pictures. Besides, we show how tensor valued connection forms act as auxiliary dynamical fields associated with the dilation, special conformal and deformation (shear) degrees of freedom, inherent to the bundle manifold. As a result, this allows to determine the bundle curvature of the theory and then to construct boundary topological invariants which give rise to a prototype (source free) gravitational Lagrangian. Finally, the Bianchi identities, the covariant field equations and the gauge currents are obtained determining completely the dynamics.
\end{abstract}

Keywords: gauge symmetry; conformal-affine Lie algebra; gravity; fiber bundle formalism

\section{INTRODUCTION}

General Relativity and Quantum Mechanics are two fundamental theories of modern physics and the Standard Model of particles is currently the most successful relativistic quantum field theory. It is a non-Abelian gauge theory (Yang-Mills theory) associated with the internal symmetry group $S U(3) \times S U(2) \times U(1)$, in which the $S U(3)$ color symmetry for the strong force in quantum chromodynamics is treated as exact whereas the $S U(2) \times U(1)$ symmetry, responsible for generating the electro-weak gauge fields, is spontaneously broken. So far as we know, there are four fundamental forces in Nature; namely, electromagnetic force, weak force, strong force and gravitational force. The Standard Model covers the first three, but not the gravitational interaction.

Here we intend to give a short summary of the various attempts to put together gravitation an the other interactions in view of a self-contained unified theory.

In General Relativity, the geometrized gravitational field is described by the metric tensor $g_{\mu \nu}$ of pseudo-Riemannian spacetime, and the field equations that the metric tensor satisfies are nonlinear. This nonlinearity is indeed a source of difficulty in quantization of General Relativity. Since the successful Standard Model of particle physics is a gauge theory in which all the fields mediating the interactions are represented by gauge potentials, a question arises to understand why the fields mediating the gravitational interaction are different from those of other fundamental forces. It is reasonable to expect that there may be a gauge theory in which the gravitational fields stand on the same footing as those of other fields. This expectation has prompted a re-examination of General Relativity from the gauge theoretical point of view.

While the gauge groups involved in the Standard Model are all internal symmetry groups (e.g. spin is an internal symmetry), the gauge groups in General Relativity must be associated to external spacetime symmetries. Therefore, the gauge theory of gravity would not be a usual Yang-Mills theory. It must be one in which gauge objects are not only the gauge potentials but also tetrads that relate the symmetry group to the external spacetime. For this reason, we have to consider a more complex nonlinear gauge theory. In General Relativity, Einstein took the spacetime metric as the basic variable representing gravity, whereas Ashtekar employed the tetrad fields and the connection forms as the fundamental variables. We also consider the tetrads and the connection forms as the fundamental fields.

R. Utiyama was the first to suggest that gravitation may be viewed as a gauge theory [1] in analogy to the Yang-Mills theory [2]. He identified the gauge potential, due to the Lorentz group, with the symmetric connection of Riemann geometry, and constructed Einstein's General Relativity as a gauge theory of the Lorentz group $S O(3,1)$ with the help of tetrad fields introduced in an ad hoc manner. Although the tetrads were necessary components of the theory to relate the Lorentz group, adopted as an internal gauge group to the external spacetime, they were not introduced as gauge fields. After, Kibble [3] constructed a gauge theory based on the Poincaré group $P(3,1)=T(3,1) \rtimes S O(3$, 1) $(\rtimes$ represents the semi-direct product) which resulted in the Einstein-Cartan theory characterized by curvature and torsion. The translation group $T(3,1)$ is considered responsible for generating the tetrads as gauge fields. Cartan 
[4] generalized the Riemann geometry to include torsion in addition to curvature. The torsion (tensor) arises from an asymmetric connection. Sciama [5], and others (R. Fikelstein [6], Hehl [7, 8]) pointed out that intrinsic spin may be the source of torsion of the underlying spacetime manifold.

Since the form and role of the tetrad fields are very different from those of gauge potentials, it has been thought that even Kibble's attempt is not satisfactory as a full gauge theory. There have been a number of gauge theories of gravitation based on several Lie groups $7,8,8,10,11,12,13]$. It was argued that a gauge theory of gravitation, corresponding to General Relativity, can be constructed with the translation group alone in the so-called teleparallel scheme. Inomata et al. 17] proposed that Kibble's gauge theory could be obtained in a way closer to the Yang-Mills approach by considering the de Sitter group $S O(4,1)$ which is reducible to the Poincaré group by group-contraction. Unlike the Poincaré group, the de Sitter group is homogeneous and the associated gauge fields are all of gauge potential type. By the Wigner-Inönu group contraction procedure, one of the five vector potentials reduces to the tetrad.

It is common to use the fiber-bundle formulation by which gauge theories can be constructed on the basis of any Lie group. A work by Hehl et al. [13] on the so-called Metric-Affine Gravity adopted, as a gauge group, the affine group $A(4, \mathbb{R})=T(4) \rtimes G L(4, \mathbb{R})$ which was realized linearly. The tetrad was identified with the nonlinearly realized translational part of the affine connection on the tangent bundle. In metric-affine gravity, the Lagrangian is quadratic in both curvature and torsion in contrast to the Einstein-Hilbert Lagrangian of General Relativity which is linear in the scalar curvature. The theory has the Einstein limit on one hand and leads to the Newtonian inverse distance potential plus the linear confinement potential in the weak field approximation on the other. This approach has been recently developed also for more general theories as $f(R)$-gravity (see [14, 15] and also [16]). As we have seen above, there are many attempts to formulate gravitation as a gauge theory. Currently no theory has been uniquely accepted as the gauge theory of gravitation.

The nonlinear approach to group realizations was originally introduced by Coleman, Wess and Zumino [18, 19] in the context of internal symmetry groups. It was later extended to the case of spacetime symmetries by Isham, Salam, and Strathdee [20, 21] considering the nonlinear action of $G L(4, \mathbb{R}) \bmod$ the Lorentz subgroup. In 1974, Borisov, Ivanov and Ogievetsky [22, 23] considered the simultaneous nonlinear realization of the affine and conformal groups. They showed that General Relativity can be viewed as a consequence of spontaneous breakdown of the affine symmetry in much the same manner that chiral dynamics in quantum chromodynamics is a result of spontaneous breakdown of chiral symmetry. In their model, gravitons are considered as Goldstone bosons associated with the affine symmetry breaking. In 1978, Chang and Mansouri [24] used the nonlinear realization scheme employing $G L(4, \mathbb{R})$ as the principal group. In 1980, Stelle and West [25] investigated the nonlinear realization induced by the spontaneous breakdown of $S O(3,2)$. In 1982 Ivanov and Niederle considered nonlinear gauge theories of the Poincaré, de Sitter, conformal and special conformal groups [26, 27]. In 1983, Ivanenko and Sardanashvily [28] considered gravity to be a spontaneously broken $G L(4, \mathbb{R})$ gauge theory. The tetrads fields arise in their formulation as a result of the reduction of the structure group of the tangent bundle from the general linear Lorentz group. In 1987, Lord and Goswami [32, 33] developed the nonlinear realization in the fiber bundle formalism based on the bundle structure $G(G / H, H)$ as suggested by Ne'eman and Regge [34]. In this approach the quotient space $G / H$ is identified with physical spacetime. Most recently, in a series of papers, A. Lopez-Pinto, J. Julve, A. Tiemblo, R. Tresguerres and E. Mielke discussed nonlinear gauge theories of gravity on the basis of the Poincaré, affine and conformal groups [36, 37, 38, 39, 41, 42].

Now, following the prescriptions of General Relativity, the physical spacetime is assumed to be a four-dimensional differential manifold. In Special Relativity, this manifold is the Minkwoski flat-spacetime $M_{4}$ while, in General Relativity, the underlying spacetime is assumed to be curved in order to describe the effects of gravitation.

As we said, Utiyama [1] proposed that General Relativity can be seen as a gauge theory based on the local Lorentz group in the same way that the Yang-Mills gauge theory [2] is developed on the basis of the internal iso-spin gauge group. In this formulation the Riemannian connection is the gravitational counterpart of the Yang-Mills gauge fields. While $S U(2)$, in the Yang-Mills theory, is an internal symmetry group, the Lorentz symmetry represents the local nature of spacetime rather than internal degrees of freedom. The Einstein Equivalence Principle, asserted for General Relativity, requires that the local spacetime structure can be identified with the Minkowski spacetime possessing Lorentz symmetry.

In order to relate local Lorentz symmetry to the external spacetime, we need to solder the local space to the external space. The soldering tools can be the tetrad fields. Utiyama regarded the tetrads as objects given a priori while they can be dynamically generated [30] and the spacetime has necessarily to be endowed with torsion in order to accommodate spinor fields. In other words, the gravitational interaction of spinning particles requires the modification of the Riemann spacetime of General Relativity to be a (non-Riemannian) curved spacetime with torsion. Although Sciama used the tetrad formalism for his gauge-like handling of gravitation, his theory fell shortcomings in treating tetrad fields as gauge fields. Following the Kibble approach [3], it can be demonstrated how gravitation can be formulated starting from a pure gauge viewpoint.

After this short summary of thirty years long attempts to put General Relativity on the same footing of non-Abelian 
gauge theories, the aim of this paper is to show, in details, how a theory of gravitation is a gauge theory which can be obtained starting from some local invariance, e.g. the local Poincaré symmetry (see [29] and references therein). This dynamical structure give rise to a gauge theory of gravity, based on a nonlinear realization of the local conformal-affine group of symmetry transformations and on conservation principles [31]. In particular, we want to show how invariance properties and conservation laws induce the gravitational field and internal (spin) fields generalizing results in [29, 40].

Specifically, in the review part of the paper, we are going to consider the general problem of how gravity, as a gauge theory, could be achieved by the nonlinear realization of the conformal-affine group. We give all the mathematical tools for this realization discussing in details the bundle approach to the gauge theories and investigating also how internal symmetries as spin could be achieved under the same standard. The result is the Invariance Induced Gravity which can be seen also as a deformation of local Poincaré Gauge Invariance. In this sense, we are going to complete the discussion in [29].

The layout of the paper is the following. In Sect.II, the standard bundle approach to the gauge theories is presented. Sect.III is devoted to the discussion of the bundle structure of gravitation, while the conformal-affine Lie algebra is introduced in Sect.IV. The group action and the bundle morphisms are discussed in Sect.V. In Sect.VI, a generalized gauge transformation law enabling the gauging of external spacetime groups is introduced. Demanding that tetrads be obtained as gauge fields requires the implementation of a nonlinear realization of the conformal-affine group. Such a nonlinear realization is carried out over the quotient space $C A(3,1) / S O(3,1)$. The covariant coset field transformations are discussed in Sect.VII In Sect.VIII, the general form of the gauge connections of the theory along with their transformation laws are obtained starting from their decomposition. Furthermore, we present the explicit structure of the conformal-affine connections. The nonlinear translational connection coefficient (transforming as a 4covector under the Lorentz group) is identified as a coframe field. After the detailed description of mathematical tools, in Sect.IX, we start with the physical realization of the approach. In particular, the tetrad components of the coframe are used, together with the Lorentz group metric, to induce the effective spacetime metric (in this sense, we can speak about an Invariance Induced Gravity). As follows in Sect.X, the bundle curvature of the theory, together with the variations of its corresponding field strength components, are determined through the Cartan structure equations. The Bianchi identities are obtained in Sect.XI. In Sect.XII, surface $(3 D)$ and bulk $(4 D)$ topological invariants are constructed. The bulk terms (obtained via exterior derivation of the surface terms) provide a means of "deriving" a prototype (source free) gravitational action (after appropriately distributing Lie star operators). The covariant field equations and gauge currents are finally obtained. Conclusions are presented in Sect. XIII.

\section{THE BUNDLE APPROACH TO THE GAUGE THEORIES}

Let us start by briefly reviewing the standard bundle approach to gauge theories. The bundle formalism, together with the conformal-affine group, will give us the mathematical tools to achieve gravity as an interaction induced from invariance properties. Besides, internal degrees of freedom of conformal-affine algebra will be related to the spin fields.

First of all, one has to verify that the usual gauge potential $\Omega$ is the pullback of connection 1-form $\omega$ by local sections of the bundle. After, the transformation laws of the $\omega$ and $\Omega$ under the action of the structure group $G$ can be deduced.

Modern formulations of gauge field theories are expressible geometrically in the language of principal fiber bundles. A fiber bundle is a structure $\langle\mathbb{P}, M, \pi ; \mathbb{F}\rangle$ where $\mathbb{P}$ (the total bundle space) and $M$ (the base space) are smooth manifolds, $\mathbb{F}$ is the fiber space and the surjection $\pi$ (a canonical projection) is a smooth map of $\mathbb{P}$ onto $M$,

$$
\pi: \mathbb{P} \rightarrow M \text {. }
$$

The inverse image $\pi^{-1}$ is diffeomorphic to $\mathbb{F}$

$$
\pi^{-1}(x) \equiv \mathbb{F}_{x} \approx \mathbb{F},
$$

and is called the fiber at $x \in M$. The partitioning $\bigcup_{x} \pi^{-1}(x)=\mathbb{P}$ is referred to as the fibration. Note that a smooth map is one whose coordinatization is $C^{\infty}$ differentiable; a smooth manifold is a space that can be covered with coordinate patches in such a manner that a change from one patch to any overlapping patch is smooth, see A. S. Schwarz [43]. Fiber bundles that admit decomposition as a direct product, locally looking like $\mathbb{P} \approx M \times \mathbb{F}$, is called trivial. Given a set of open coverings $\left\{\mathcal{U}_{i}\right\}$ of $M$ with $x \in\left\{\mathcal{U}_{i}\right\} \subset M$ satisfying $\bigcup_{\alpha} \mathcal{U}_{\alpha}=M$, the diffeomorphism map is given by

$$
\chi_{i}: \mathcal{U}_{i} \times_{M} G \rightarrow \pi^{-1}\left(\mathcal{U}_{i}\right) \in \mathbb{P}
$$

$\left(\times_{M}\right.$ represents the fiber product of elements defined over space $\left.M\right)$ such that $\pi\left(\chi_{i}(x, g)\right)=x$ and $\chi_{i}(x, g)=$ $\chi_{i}\left(x,(i d)_{G}\right) g=\chi_{i}(x) g \forall x \in\left\{\mathcal{U}_{i}\right\}$ and $g \in G$. Here, $(i d)_{G}$ represents the identity element of group $G$. In order to 
obtain the global bundle structure, the local charts $\chi_{i}$ must be glued together continuously. Consider two patches $\mathcal{U}_{n}$ and $\mathcal{U}_{m}$ with a non-empty intersection $\mathcal{U}_{n} \cap \mathcal{U}_{m} \neq \emptyset$. Let $\rho_{n m}$ be the restriction of $\chi_{n}^{-1}$ to $\pi^{-1}\left(\mathcal{U}_{n} \cap \mathcal{U}_{m}\right)$ defined by $\rho_{n m}: \pi^{-1}\left(\mathcal{U}_{n} \cap \mathcal{U}_{m}\right) \rightarrow\left(\mathcal{U}_{n} \cap \mathcal{U}_{m}\right) \times_{M} G_{n}$. Similarly let $\rho_{m n}: \pi^{-1}\left(\mathcal{U}_{m} \cap \mathcal{U}_{n}\right) \rightarrow\left(\mathcal{U}_{m} \cap \mathcal{U}_{n}\right) \times_{M} G_{m}$ be the restriction of $\chi_{m}^{-1}$ to $\pi^{-1}\left(\mathcal{U}_{n} \cap \mathcal{U}_{m}\right)$. The composite diffeomorphism $\Lambda_{n m} \in G$

$$
\Lambda_{m n}:\left(\mathcal{U}_{n} \cap \mathcal{U}_{m}\right) \times G_{n} \rightarrow\left(\mathcal{U}_{m} \cap \mathcal{U}_{n}\right) \times_{M} G_{m}
$$

defined as

$$
\Lambda_{i j}(x) \equiv \rho_{j i} \circ \rho_{i j}^{-1}=\chi_{i, x} \circ \chi_{j, x}^{-1}: \mathbb{F} \rightarrow \mathbb{F}
$$

constitute the transition function between bundle charts $\rho_{n m}$ and $\rho_{m n}$ (o represents the group composition operation) where the diffeomorphism $\chi_{i, x}: \mathbb{F} \rightarrow \mathbb{F}_{x}$ is written as $\chi_{i, x}(g):=\chi_{i}(x, g)$ and satisfies $\chi_{j}(x, g)=\chi_{i}\left(x, \Lambda_{i j}(x) g\right)$. The transition functions $\left\{\Lambda_{i j}\right\}$ can be interpreted as passive gauge transformations. They satisfy the identity $\Lambda_{i i}(x)$, inverse $\Lambda_{i j}(x)=\Lambda_{j i}^{-1}(x)$ and cocycle $\Lambda_{i j}(x) \Lambda_{j k}(x)=\Lambda_{i k}(x)$ consistency conditions. For trivial bundles, the transition function reduces to

$$
\Lambda_{i j}(x)=g_{i}^{-1} g_{j}
$$

where $g_{i}: \mathbb{F} \rightarrow \mathbb{F}$ is defined by $g_{i}:=\chi_{i, x}^{-1} \circ \tilde{\chi}_{i, x}$ provided the local trivializations $\left\{\chi_{i}\right\}$ and $\left\{\tilde{\chi}_{i}\right\}$ give rise to the same fiber bundle.

A section is defined as a smooth map

$$
s: M \rightarrow \mathbb{P}
$$

such that $s(x) \in \pi^{-1}(x)=\mathbb{F}_{x} \forall x \in M$ and satisfies

$$
\pi \circ s=(i d)_{M}
$$

where $(i d)_{M}$ is the identity element of $M$. It assigns to each point $x \in M$ a point in the fiber over $x$. Trivial bundles admit global sections.

A bundle is a principal fiber bundle $\langle\mathbb{P}, \mathbb{P} / G, G, \pi\rangle$ provided the Lie group $G$ acts freely (i.e. if $p g=p$ then $\left.g=(i d)_{G}\right)$ on $\mathbb{P}$ to the right $R_{g} p=p g, p \in \mathbb{P}$, preserves fibers on $\mathbb{P}\left(R_{g}: \mathbb{P} \rightarrow \mathbb{P}\right)$, and is transitive on fibers. Furthermore, there must exist local trivializations compatible with the $G$ action. Hence, $\pi^{-1}\left(\mathcal{U}_{i}\right)$ is homeomorphic to $\mathcal{U}_{i} \times{ }_{M} G$ and the fibers of $\mathbb{P}$ are diffeomorphic to $G$. The trivialization or inverse diffeomorphism map is given by

$$
\chi_{i}^{-1}: \pi^{-1}\left(\mathcal{U}_{i}\right) \rightarrow \mathcal{U}_{i} \times{ }_{M} G
$$

such that $\chi^{-1}(p)=(\pi(p), \varphi(p)) \in \mathcal{U}_{i} \times_{M} G, p \in \pi^{-1}\left(\mathcal{U}_{i}\right) \subset \mathbb{P}$, where we see from the above definition that $\varphi$ is a local mapping of $\pi^{-1}\left(\mathcal{U}_{i}\right)$ into $G$ satisfying $\varphi\left(L_{g} p\right)=\varphi(p) g$ for any $p \in \pi^{-1}(\mathcal{U})$ and any $g \in G$. Observe that the elements of $\mathbb{P}$ which are projected onto the same $x \in\left\{\mathcal{U}_{i}\right\}$ are transformed into one another by the elements of $G$. In other words, the fibers of $\mathbb{P}$ are the orbits of $G$ and at the same time, the set of elements which are projected onto the same $x \in \mathcal{U} \subset M$. This observation motivates calling the action of the group vertical and the base manifold horizontal. The diffeomorphism map $\chi_{i}$ is called the local gauge since $\chi_{i}^{-1}$ maps $\pi^{-1}\left(\mathcal{U}_{i}\right)$ onto the direct (Cartesian) product $\mathcal{U}_{i} \times_{M} G$. The action $L_{g}$ of the structure group $G$ on $\mathbb{P}$ defines an isomorphism of the Lie algebra $\mathfrak{g}$ of $G$ onto the Lie algebra of vertical vector fields on $\mathbb{P}$ tangent to the fiber at each $p \in \mathbb{P}$ called fundamental vector fields

$$
\lambda_{g}: T_{p}(\mathbb{P}) \rightarrow T_{g p}(\mathbb{P})=T_{\pi(p)}(\mathbb{P}),
$$

where $T_{p}(\mathbb{P})$ is the space of tangents at $p$, i.e. $T_{p}(\mathbb{P}) \in T(\mathbb{P})$. The map $\lambda$ is a linear isomorphism for every $p \in \mathbb{P}$ and is invariant with respect to the action of $G$, that is, $\lambda_{g}:\left(\lambda_{g *} T_{p}(\mathbb{P})\right) \rightarrow T_{g p}(\mathbb{P})$, where $\lambda_{g *}$ is the differential push forward map induced by $\lambda_{g}$ defined by $\lambda_{g *}: T_{p}(\mathbb{P}) \rightarrow T_{g p}(\mathbb{P})$.

Since the principal bundle $\mathbb{P}(M, G)$ is a differentiable manifold, we can define tangent $T(\mathbb{P})$ and cotangent $T^{*}(\mathbb{P})$ bundles. The tangent space $T_{p}(\mathbb{P})$ defined at each point $p \in \mathbb{P}$ may be decomposed into a vertical $V_{p}(\mathbb{P})$ and horizontal $H_{p}(\mathbb{P})$ subspace as $T_{p}(\mathbb{P}):=V_{p}(\mathbb{P}) \oplus H_{p}(\mathbb{P})$ (where $\oplus$ represents the direct sum). The space $V_{p}(\mathbb{P})$ is a subspace of $T_{p}(\mathbb{P})$ consisting of all tangent vectors to the fiber passing through $p \in \mathbb{P}$, and $H_{p}(\mathbb{P})$ is the subspace complementary to $V_{p}(\mathbb{P})$ at $p$. The vertical subspace $V_{p}(\mathbb{P}):=\left\{X \in T(\mathbb{P}) \mid \pi(X) \in \mathcal{U}_{i} \subset M\right\}$ is uniquely determined by the structure of $\mathbb{P}$, whereas the horizontal subspace $H_{p}(\mathbb{P})$ cannot be uniquely specified. Thus we require the following condition: when $p$ transforms as $p \rightarrow p^{\prime}=p g, H_{p}(\mathbb{P})$ transforms as [44],

$$
R_{g *} H_{p}(\mathbb{P}) \rightarrow H_{p^{\prime}}(\mathbb{P})=R_{g} H_{p}(\mathbb{P})=H_{p g}(\mathbb{P}) .
$$


Let the local coordinates of $\mathbb{P}(M, G)$ be $p=(x, g)$ where $x \in M$ and $g \in G$. Let $\mathbf{G}_{A}$ denote the generators of the Lie algebra $\mathfrak{g}$ corresponding to group $G$ satisfying the commutators $\left[\mathbf{G}_{A}, \mathbf{G}_{B}\right]=f_{A B}{ }^{C} \mathbf{G}_{C}$, where $f_{A B}{ }^{C}$ are the structure constants of $G$. Let $\Omega$ be a connection form defined by $\Omega^{A}:=\Omega_{i}^{A} d x^{i} \in \mathfrak{g}$. Let $\omega$ be a connection 1-form defined by

$$
\omega:=\widetilde{g}^{-1} \pi_{\mathbb{P} M}^{*} \Omega \widetilde{g}+\widetilde{g}^{-1} d \widetilde{g}
$$

(* represents the differential pullback map) belonging to $\mathfrak{g} \otimes T_{p}^{*}(\mathbb{P})$ where $T_{p}^{*}(\mathbb{P})$ is the space dual to $T_{p}(\mathbb{P})$. The differential pullback map applied to a test function $\varphi$ and $p$-forms $\alpha$ and $\beta$ satisfy $f^{*} \varphi=\varphi \circ f,(g \circ f)^{*}=f^{*} g^{*}$ and $f^{*}(\alpha \wedge \beta)=f^{*} \alpha \wedge f^{*} \beta$. If $G$ is represented by a $d$-dimensional $d \times d$ matrix, then $\mathbf{G}_{A}=\left[\mathbf{G}_{\alpha \beta}\right], \widetilde{g}=\left[\widetilde{g}^{\alpha \beta}\right]$, where $\alpha, \beta=1,2,3, \ldots d$. Thus, $\omega$ assumes the form

$$
\omega_{\alpha}^{\beta}=\left(\widetilde{g}^{-1}\right)_{\alpha \gamma} d \widetilde{g}^{\gamma \beta}+\left(\widetilde{g}^{-1}\right)_{\rho \gamma} \pi_{\mathbb{P} M}^{*} \Omega_{\sigma i}^{\rho} \mathbf{G}_{\alpha}^{\gamma} \widetilde{g}^{\sigma \beta} \otimes d x^{i} .
$$

If $M$ is $n$-dimensional, the tangent space $T_{p}(\mathbb{P})$ is $(n+d)$-dimensional. Since the vertical subspace $V_{p}(\mathbb{P})$ is tangential to the fiber $G$, it is $d$-dimensional. Accordingly, $H_{p}(\mathbb{P})$ is $n$-dimensional. The basis of $V_{p}(\mathbb{P})$ can be taken to be $\partial_{\alpha \beta}:=\frac{\partial}{\partial g^{\alpha \beta}}$. Now, let the basis of $H_{p}(\mathbb{P})$ be denoted by

$$
E_{i}:=\partial_{i}+\Gamma_{i}^{\alpha \beta} \partial_{\alpha \beta}, i=1,2,3, . . n \text { and } \alpha, \beta=1,2,3, . . d
$$

where $\partial_{i}=\frac{\partial}{\partial x^{2}}$. The connection 1-form $\omega$ projects $T_{p}(\mathbb{P})$ onto $V_{p}(\mathbb{P})$. In order for $X \in T_{p}(\mathbb{P})$ to belong to $H_{p}(\mathbb{P})$, that is for $X \in H_{p}(\mathbb{P}), \omega_{p}(X)=\langle\omega(p) \mid X\rangle=0$. In other words,

$$
H_{p}(\mathbb{P}):=\left\{X \in T_{p}(\mathbb{P}) \mid \omega_{p}(X)=0\right\},
$$

from which $\Omega_{i}^{\alpha \beta}$ can be determined. The inner product appearing in $\omega_{p}(X)=\langle\omega(p) \mid X\rangle=0$ is a map $\langle\cdot \mid \cdot\rangle$ : $T_{p}^{*}(\mathbb{P}) \times T_{p}(\mathbb{P}) \rightarrow \mathbb{R}$ defined by $\langle W \mid V\rangle=W_{\mu} V^{\nu}\left\langle d x^{\mu} \mid \frac{\partial}{\partial x^{\nu}}\right\rangle=W_{\mu} V^{\nu} \delta_{\nu}^{\mu}$, where the 1-form $W$ and vector $V$ are given by $W=W_{\mu} d x^{\mu}$ and $V=V^{\mu} \frac{\partial}{\partial x^{\nu}}$. Observe also that, $\left\langle d g^{\alpha \beta} \mid \partial_{\rho \sigma}\right\rangle=\delta_{\rho}^{\alpha} \delta_{\sigma}^{\beta}$.

We parameterize an arbitrary group element $\widetilde{g}_{\lambda}$ as $\widetilde{g}(\lambda)=e^{\lambda^{A} \mathbf{G}_{A}}=e^{\lambda \cdot \mathbf{G}}, A=1, . . \operatorname{dim}(\mathfrak{g})$. The right action $R_{\widetilde{g}(\lambda)}=R_{\exp (\lambda \cdot G)}$ on $p \in \mathbb{P}$, i.e. $R_{\exp (\lambda \cdot \mathbf{G})} p=p \exp (\lambda \cdot \mathbf{G})$, defines a curve through $p$ in $\mathbb{P}$. Define a vector $G^{\#} \in T_{p}(\mathbb{P})$ by $[44]$

$$
G^{\#} f(p):=\left.\frac{d}{d t} f(p \exp (\lambda \cdot \mathbf{G}))\right|_{\lambda=0}
$$

where $f: \mathbb{P} \rightarrow \mathbb{R}$ is an arbitrary smooth function. Since the vector $G^{\#}$ is tangent to $\mathbb{P}$ at $p, G^{\#} \in V_{p}(\mathbb{P})$, the components of the vector $G^{\#}$ are the fundamental vector fields at $p$ which constitute $V(\mathbb{P})$. The components of $G^{\#}$ may also be viewed as a basis element of the Lie algebra $\mathfrak{g}$. Given $G^{\#} \in V_{p}(\mathbb{P}), \mathbf{G} \in \mathfrak{g}$,

$$
\begin{aligned}
\omega_{p}\left(G^{\#}\right) & =\left\langle\omega(p) \mid G^{\#}\right\rangle=\widetilde{g}^{-1} d \widetilde{g}\left(G^{\#}\right)+\widetilde{g}^{-1} \pi_{\mathbb{P} M}^{*} \Omega \widetilde{g}\left(G^{\#}\right) \\
& =\left.\widetilde{g}_{p}^{-1} \widetilde{g}_{p} \frac{d}{d \lambda}(\exp (\lambda \cdot \mathbf{G}))\right|_{\lambda=0},
\end{aligned}
$$

where use was made of $\pi_{\mathbb{P} M *} G^{\#}=0$. Hence, $\omega_{p}\left(G^{\#}\right)=\mathbf{G}$. An arbitrary vector $X \in H_{p}(\mathbb{P})$ may be expanded in a basis spanning $H_{p}(\mathbb{P})$ as $X:=\beta^{i} E_{i}$. By direct computation, one can show

$$
\left\langle\omega_{\alpha}^{\beta} \mid X\right\rangle=\left(\widetilde{g}^{-1}\right)_{\alpha \gamma} \beta^{i} \Gamma_{i}^{\gamma \beta}+\left(\widetilde{g}^{-1}\right)_{\alpha \gamma} \pi_{\mathbb{P} M}^{*} \Omega^{\rho}{ }_{\sigma i} \beta^{i} \mathbf{G}_{\rho}^{\gamma} \widetilde{g}^{\sigma \beta}=0, \forall \beta^{i}
$$

Equation (18) yields

$$
\left(\widetilde{g}^{-1}\right)_{\alpha \gamma} \Gamma_{i}^{\gamma \beta}+\left(\widetilde{g}^{-1}\right)_{\alpha \gamma} \pi_{\mathbb{P} M}^{*} \Omega_{\sigma i}^{\rho} \mathbf{G}_{\rho}^{\gamma} \widetilde{g}^{\sigma \beta}=0
$$

from which we obtain

$$
\Gamma_{i}^{\gamma \beta}=-\pi_{\mathbb{P} M}^{*} \Omega^{\rho}{ }_{\sigma i} \mathbf{G}_{\rho}^{\gamma} \widetilde{g}^{\sigma \beta} .
$$

In this manner, the horizontal component is completely determined. An arbitrary tangent vector $\mathfrak{X} \in T_{p}(\mathbb{P})$ defined at $p \in \mathbb{P}$ takes the form

$$
\mathfrak{X}=A^{\alpha \beta} \partial_{\alpha \beta}+B^{i}\left(\partial_{i}-\pi_{\mathbb{P} M}^{*} \Omega^{\rho}{ }_{\sigma i} \mathbf{G}_{\rho}^{\alpha} \widetilde{g}^{\sigma \beta} \partial_{\alpha \beta}\right),
$$


where $A^{\alpha \beta}$ and $B^{i}$ are constants. The vector field $\mathfrak{X}$ is comprised of horizontal $\mathfrak{X}^{H}:=B^{i}\left(\partial_{i}-\pi_{\mathbb{P} M}^{*} \Omega^{\rho}{ }_{\sigma i} \mathbf{G}_{\rho}^{\alpha} \widetilde{g}^{\sigma \beta} \partial_{\alpha \beta}\right) \in$ $H(\mathbb{P})$ and vertical $\mathfrak{X}^{V}:=A^{\alpha \beta} \partial_{\alpha \beta} \in V(\mathbb{P})$ components.

Let $\mathfrak{X} \in T_{p}(\mathbb{P})$ and $g \in \mathbf{G}$, then

$$
R_{g}^{*} \omega(\mathfrak{X})=\omega\left(R_{g *} \mathfrak{X}\right)=\widetilde{g}_{p g}^{-1} \Omega\left(R_{g *} \mathfrak{X}\right) \widetilde{g}_{p g}+\widetilde{g}_{p g}^{-1} d \widetilde{g}_{p g}\left(R_{g *} \mathfrak{X}\right),
$$

Observing that $\widetilde{g}_{p g}=\widetilde{g}_{p} g$ and $\widetilde{g}_{g p}^{-1}=g^{-1} \widetilde{g}_{p}^{-1}$ the first term on the RHS of (22) reduces to $\widetilde{g}_{p g}^{-1} \Omega\left(R_{g *} \mathfrak{X}\right) \widetilde{g}_{p g}=$ $g^{-1} \widetilde{g}_{p}^{-1} \Omega\left(R_{g *} \mathfrak{X}\right) \widetilde{g}_{p} g$ while the second term gives $\widetilde{g}_{p g}^{-1} d \widetilde{g}_{p g}\left(R_{g *} \mathfrak{X}\right)=g^{-1} \widetilde{g}_{p}^{-1} d\left(R_{g *} \mathfrak{X}\right) \widetilde{g}_{p} g$. We therefore conclude

$$
R_{g}^{*} \omega_{\lambda}=a d_{g^{-1}} \omega_{\lambda}
$$

where the adjoint map ad is defined by

$$
a d_{g} Y:=L_{g *} \circ R_{g^{-1 *}} \circ Y=g Y g^{-1}, a d_{g^{-1}} Y:=g^{-1} Y g .
$$

The potential $\Omega^{A}$ can be obtained from $\omega$ as $\Omega^{A}=s^{*} \omega$. To demonstrate this, let $Y \in T_{p}(M)$ and $\widetilde{g}$ be specified by the inverse diffeomorphism or trivialization map (9) with $\chi_{\lambda}^{-1}(p)=\left(x, \widetilde{g}_{\lambda}\right)$ for $p(x)=s_{\lambda}(x) \cdot \widetilde{g}_{\lambda}$. We find $s_{i}^{*} \omega(Y)=\widetilde{g}^{-1} \Omega\left(\pi_{*} s_{i *} Y\right) \widetilde{g}+\widetilde{g}^{-1} d \widetilde{g}\left(s_{i *} Y\right)$, where we [44] have used $s_{i *} Y \in T_{s_{i}}(\mathbb{P}), \pi_{*} s_{i *}=(i d)_{T_{p}(M)}$ and $\widetilde{g}=(i d)_{G}$ at $s_{i}$ implying $\widetilde{g}^{-1} d \widetilde{g}\left(s_{i *} Y\right)=0$. Hence,

$$
s_{i}^{*} \omega(Y)=\Omega(Y) .
$$

To determine the gauge transformation of the connection 1-form $\omega$ we use the fact that $R_{\widetilde{g} *} X=X \widetilde{g}$ for $X \in T_{p}(M)$ and the transition functions $\widetilde{g}_{n m} \in G$ defined between neighboring bundle charts (6). By direct computation we get

$$
\begin{aligned}
c_{j *} X & =\left.\frac{d}{d t} c_{j}(\lambda(t))\right|_{t=0}=\left.\frac{d}{d t}\left[c_{i}(\lambda(t)) \cdot \widetilde{g}_{i j}\right]\right|_{t=0} \\
& =R_{\widetilde{g}_{i j} *} c_{i}^{*}(X)+\left(\widetilde{g}_{j i}^{-1}(x) d \widetilde{g}_{i j}(X)\right)^{\#} .
\end{aligned}
$$

where $\lambda(t)$ is a curve in $M$ with boundary values $\lambda(0)=m$ and $\left.\frac{d}{d t} \lambda(t)\right|_{t=0}=X$. Thus, we obtain the useful result

$$
c_{*} X=R_{\widetilde{g} *}\left(c_{*} X\right)+\left(\widetilde{g}^{-1} d \widetilde{g}(X)\right)^{\#} .
$$

Applying $\omega$ to (27) we get

$$
\omega\left(c_{*} X\right)=c^{*} \omega(X)=a d_{\widetilde{g}^{-1}} c^{*} \omega(X)+\widetilde{g}^{-1} d \widetilde{g}(X), \forall X .
$$

Hence, the gauge transformation of the local gauge potential $\Omega$ reads,

$$
\Omega \rightarrow \Omega^{\prime}=a d_{\widetilde{g}^{-1}}(d+\Omega)=\widetilde{g}^{-1}(d+\Omega) \widetilde{g} .
$$

Since $\Omega=c^{*} \omega$ we obtain from (29) the gauge transformation law of $\omega$

$$
\omega \rightarrow \omega^{\prime}=\widetilde{g}^{-1}(d+\omega) \widetilde{g} .
$$

Now we are ready to undertake the task to construct the bundle structure of gravitation.

\section{THE GENERALIZED BUNDLE STRUCTURE OF GRAVITATION}

Let us recall the definition of gauge transformations in the context of ordinary fiber bundles. This step will be extremely relevant to induce metric and dynamics from invariance properties. Given a principal fiber bundle $\mathbb{P}(M$, $G ; \pi)$ with base space $M$ and standard $G$-diffeomorphic fiber, gauge transformations are characterized by bundle isomorphisms [46] $\lambda: \mathbb{P} \rightarrow \mathbb{P}$ exhausting all diffeomorphisms $\lambda_{M}$ on $M$. This mapping is called an automorphism of $\mathbb{P}$ provided it is equivariant with respect to the action of $G$. This amounts to restricting the action $\lambda$ of $G$ along local fibers leaving the base space unaffected. Indeed, with regard to gauge theories of internal symmetry groups, a gauge transformation is a fiber preserving bundle automorphism, i.e. diffeomorphisms $\lambda$ with $\lambda_{M}=(i d)_{M}$. The automorphisms $\lambda$ form a group called the automorphism group $A u t \mathbb{P}$ of $\mathbb{P}$. The gauge transformations form a subgroup of $A u t_{\mathbb{P}}$ called the gauge group $G\left(A u t_{\mathbb{P}}\right)$ (or $G$ in short) of $\mathbb{P}$. 
The map $\lambda$ is required to satisfy two conditions, namely its commutability with the right action of $G$ [the equivariance condition $\left.\lambda\left(R_{g}(p)\right)=\lambda(p g)=\lambda(p) g\right]$

$$
\lambda \circ R_{g}(p)=R_{g}(p) \circ \lambda, \quad p \in \mathbb{P}, g \in G
$$

according to which fibers are mapped into fibers, and the verticality condition

$$
\pi \circ \lambda(u)=\pi(u)
$$

where $u$ and $\lambda(u)$ belong to the same fiber. The last condition ensures that no diffeomorphisms $\lambda_{M}: M \rightarrow M$ given by

$$
\lambda_{M} \circ \pi(u)=\pi \circ \lambda(u),
$$

be allowed on the base space $M$. In a gauge description of gravitation, one is interested in gauging external transformation groups. That is to say the group action on spacetime coordinates cannot be neglected. The spaces of internal fiber and external base must be interlocked in the sense that transformations in one space must induce corresponding transformations in the other. The usual definition of a gauge transformation, i.e. as a displacement along local fibers not affecting the base space, must be generalized to reflect this interlocking. One possible way of framing this interlocking is to employ a nonlinear realization of the gauge group $G$, provided a closed subgroup $H \subset G$ exist. The interlocking requirement is then transformed into the interplay between groups $G$ and one of its closed subgroups $H$.

Denote by $G$ a Lie group with elements $\{g\}$. Let $H$ be a closed subgroup of $G$ specified by [37, 67]

$$
H:=\left\{h \in G \mid \Pi\left(R_{h} g\right)=\pi(g), \forall g \in G\right\},
$$

with elements $\{h\}$ and known linear representations $\rho(h)$. Here $\Pi$ is the first of the two projection maps in (37), and $R_{h}$ is the right group action. Let $M$ be a differentiable manifold with points $\{x\}$ to which $G$ and $H$ may be referred, i.e. $g=g(x)$ and $h=h(x)$. Being that $G$ and $H$ are Lie groups, they are also manifolds. The right action of $H$ on $G$ induce a complete partition of $G$ into mutually disjoint orbits $g H$. Since $g=g(x)$, all elements of $g H=\left\{g h_{1}, g h_{2}, g h_{3}, \cdots, g h_{n}\right\}$ are defined over the same $x$. Thus, each orbit $g H$ constitute an equivalence class of point $x$, with equivalence relation $g \equiv g^{\prime}$ where $g^{\prime}=R_{h} g=g h$. By projecting each equivalence class onto a single element of the quotient space $\mathcal{M}:=G / H$, the group $G$ becomes organized as a fiber bundle in the sense that $G=\bigcup_{i}\left\{g_{i} H\right\}$. In this manner the manifold $G$ is viewed as a fiber bundle $G(\mathcal{M}, H ; \Pi)$ with $H$-diffeomorphic fibers $\Pi^{-1}(\xi): G \rightarrow \mathcal{M}=g H$ and base space $\mathcal{M}$. A composite principal fiber bundle $\mathbb{P}(M, G ; \pi)$ is one whose $G$-diffeomorphic fibers possess the fibered structure $G(\mathcal{M}, H ; \Pi) \simeq \mathcal{M} \times H$ described above. The bundle $\mathbb{P}$ is then locally isomorphic to $M \times G(\mathcal{M}, H)$. Moreover, since an element $g \in G$ is locally homeomorphic to $\mathcal{M} \times H$ the elements of $\mathbb{P}$ are - by transitivity - also locally homeomorphic to $M \times \mathcal{M} \times H \simeq \Sigma \times H$ where (locally) $\Sigma \simeq M \times \mathcal{M}$. Thus, an alternative view [39] of $\mathbb{P}(M, G ; \pi)$ is provided by the $\mathbb{P}$-associated $H$-bundle $\mathbb{P}(\Sigma, H ; \widetilde{\pi})$. The total space $\mathbb{P}$ may be regarded as $G(\mathcal{M}, H ; \Pi)$-bundles over base space $M$ or equivalently as $H$-fibers attached to manifold $\Sigma \simeq M \times \mathcal{M}$.

The nonlinear realization technique [18, 19] provides a way to determine the transformation properties of fields defined on the quotient space $G / H$. The nonlinear realization of $\operatorname{Diff}(4, \mathbb{R})$ becomes tractable due to a theorem given by V. I. Ogievetsky. According to the Ogievetsky theorem [22], the algebra of the infinite dimensional group $\operatorname{Diff}(4, \mathbb{R})$ can be taken as the closure of the finite dimensional algebras of $S O(4,2)$ and $A(4, \mathbb{R})$. Remind that the Lorentz group generates transformations that preserve the quadratic form on Minkowski spacetime built from the metric tensor, while the special conformal group generates infinitesimal angle-preserving transformations on Minkowski spacetime. The affine group is a generalization of the Poincaré group where the Lorentz group is replaced by the group of general linear transformations [29]. As such, the affine group generates translations, Lorentz transformations, volume preserving shear and volume changing dilation transformations. As a consequence, the nonlinear realization of $\operatorname{Diff}(4, \mathbb{R}) / S O(3,1)$ can be constructed by taking a simultaneous realization of the conformal group $S O(4,2)$ and the affine group $A(4, \mathbb{R}):=\mathbb{R}^{4} \rtimes G L(4, \mathbb{R})$ on the coset spaces $A(4, \mathbb{R}) / S O(3,1)$ and $S O(4,2) / S O(3,1)$. One possible interpretation of this theorem is that the conform-affine group (defined below) may be the largest subgroup of $\operatorname{Diff}(4, \mathbb{R})$ whose transformations may be put into the form of a generalized coordinate transformation. We remark that a nonlinear realization can be made linear by embedding the representation in a sufficiently higher dimensional space. Alternatively, a linear group realization becomes nonlinear when subject to constraints. One type of relevant constraints may be those responsible for symmetry reduction from $\operatorname{Diff}(4, \mathbb{R})$ to $S O(3,1)$ for instance.

We take the group $C A(3,1)$ as the basic symmetry group $G$. The conformal-affine group consists of the groups $S O(4,2)$ and $A(4, \mathbb{R})$. In particular, conformal-affine is proportional to the union $S O(4,2) \cup A(4, \mathbb{R})$. We know however (see section Conform-Affine Lie Algebra) that the affine and special conformal groups have several group generators in common. These common generators reside in the intersection $S O(4,2) \cap A(4, \mathbb{R})$ of the two groups, 
within which there are two copies of $\Pi:=D \times P(3,1)$, where $D$ is the group of scale transformations (dilations) and $P(3,1):=T(3,1) \rtimes S O(3,1)$ is the Poincaré group. We define the conformal-affine group as the union of the affine and conformal groups minus one copy of the overlap $\Pi$, i.e. $C A(3,1):=S O(4,2) \cup A(4, \mathbb{R})-\Pi$. Being defined in this way we recognize that $C A(3,1)$ is a 24 parameter Lie group representing the action of Lorentz transformations (6), translations (4), special conformal transformations (4), spacetime shears (9) and scale transformations (1). In this paper, we are obtaining the nonlinear realization of $C A(3,1)$ modulo $S O(3,1)$.

\section{THE CONFORMAL-AFFINE LIE ALGEBRA}

In order to implement the nonlinear realization procedure, we choose the partition Diff( 4 , $\mathbb{R})$ with respect to the Lorentz group. By Ogievetsky's theorem [22], we identify representations of Diff(4, $\mathbb{R}) / S O(3,1)$ with those of $C A(3$, 1) $/ S O(3,1)$. The 20 generators of affine transformations can be decomposed into the 4 translational $\mathbf{P}_{\mu}^{\text {Aff }}$ and 16 $G L(4, \mathbb{R})$ transformations $\boldsymbol{\Lambda}_{\alpha}^{\beta}$. The 16 generators $\boldsymbol{\Lambda}_{\alpha}^{\beta}$ may be further decomposed into the 6 Lorentz generators $\mathbf{L}_{\alpha}^{\beta}$ plus the remaining 10 generators of symmetric linear transformation $\mathbf{S}_{\alpha}^{\beta}$, that is, $\boldsymbol{\Lambda}_{\beta}^{\alpha}=\mathbf{L}_{\beta}^{\alpha}+\mathbf{S}_{\beta}^{\alpha}$. The 10 parameter symmetric linear generators $\mathbf{S}_{\alpha}^{\beta}$ can be factored into the 9 parameter shear (the traceless part of $\mathbf{S}_{\alpha}^{\beta}$ ) generator defined by ${ }^{\dagger} \mathbf{S}_{\alpha}{ }_{\alpha}=\mathbf{S}_{\alpha}{ }^{\beta}-\frac{1}{4} \delta_{\alpha}{ }^{\beta} \mathbf{D}$, and the 1 parameter dilaton generator $\mathbf{D}=\operatorname{tr}\left(\mathbf{S}_{\alpha}{ }^{\beta}\right)$. Shear transformations generated by ${ }^{\dagger} \mathbf{S}_{\alpha}^{\beta}$ describe shape changing, volume preserving deformations, while the dilaton generator gives rise to volume changing transformations. The four diagonal elements of $\mathbf{S}_{\alpha}^{\beta}$ correspond to the generators of projective transformations. The 15 generators of conformal transformations are defined in terms of the set $\left\{J_{A B}\right\}$ where $A=0,1,2, . .5$. The elements $J_{A B}$ can be decomposed into translations $\mathbf{P}_{\mu}^{\text {Conf }}:=J_{5 \mu}+J_{6 \mu}$, special conformal generators $\boldsymbol{\Delta}_{\mu}:=J_{5 \mu}-J_{6 \mu}$, dilatons $\mathbf{D}:=J_{56}$ and the Lorentz generators $\mathbf{L}_{\alpha \beta}:=J_{\alpha \beta}$. The Lie algebra of $C A(3,1)$ is characterized by the commutation relations

$$
\begin{gathered}
{\left[\boldsymbol{\Lambda}_{\alpha \beta}, \mathbf{D}\right]=\left[\boldsymbol{\Delta}_{\alpha}, \boldsymbol{\Delta}_{\beta}\right]=0,\left[\mathbf{P}_{\alpha}, \mathbf{P}_{\beta}\right]=[\mathbf{D}, \mathbf{D}]=0,} \\
{\left[\mathbf{L}_{\alpha \beta}, \mathbf{P}_{\mu}\right]=i o_{\mu[\alpha} \mathbf{P}_{\beta]},\left[\mathbf{L}_{\alpha \beta}, \boldsymbol{\Delta}_{\gamma}\right]=i o_{[\alpha \mid \gamma} \boldsymbol{\Delta}_{\mid \beta]},} \\
{\left[\boldsymbol{\Lambda}_{\beta}^{\alpha}, \mathbf{P}_{\mu}\right]=i \delta_{\mu}^{\alpha} \mathbf{P}_{\beta},\left[\boldsymbol{\Lambda}_{\beta}^{\alpha}, \boldsymbol{\Delta}_{\mu}\right]=i \delta_{\mu}^{\alpha} \boldsymbol{\Delta}_{\beta},} \\
{\left[\mathbf{S}_{\alpha \beta}, \mathbf{P}_{\mu}\right]=i o_{\mu(\alpha} \mathbf{P}_{\beta)},\left[\mathbf{P}_{\alpha}, \mathbf{D}\right]=-i \mathbf{P}_{\alpha}} \\
{\left[\mathbf{L}_{\alpha \beta}, \mathbf{L}_{\mu \nu}\right]=-i\left(o_{\alpha[\mu} \mathbf{L}_{\nu] \beta}-o_{\beta[\mu} \mathbf{L}_{\nu] \alpha}\right)} \\
{\left[\mathbf{S}_{\alpha \beta}, \mathbf{S}_{\mu \nu}\right]=i\left(o_{\alpha(\mu} \mathbf{L}_{\nu) \beta}-o_{\beta(\mu} \mathbf{L}_{\nu) \alpha}\right)} \\
{\left[\mathbf{L}_{\alpha \beta}, \mathbf{S}_{\mu \nu}\right]=i\left(o_{\alpha(\mu} \mathbf{S}_{\nu) \beta}-o_{\beta(\mu} \mathbf{S}_{\nu) \alpha}\right)} \\
{\left[\boldsymbol{\Delta}_{\alpha}, \mathbf{D}\right]=i \boldsymbol{\Delta}_{\alpha},\left[\mathbf{S}_{\mu \nu}, \boldsymbol{\Delta}_{\alpha}\right]=i o_{\alpha(\mu} \boldsymbol{\Delta}_{\nu)}} \\
{\left[\boldsymbol{\Lambda}_{\beta}^{\alpha}, \boldsymbol{\Lambda}_{\nu}^{\mu}\right]=i\left(\delta_{\nu}^{\alpha} \boldsymbol{\Lambda}_{\beta}^{\mu}-\delta_{\beta}^{\mu} \boldsymbol{\Lambda}_{\nu}^{\alpha}\right)} \\
{\left[\mathbf{P}_{\alpha}, \boldsymbol{\Delta}_{\beta}\right]=2 i\left(o_{\alpha \beta} \mathbf{D}-\mathbf{L}_{\alpha \beta}\right)}
\end{gathered}
$$

where $o_{\alpha \beta}=\operatorname{diag}(-1,1,1,1)$ is Lorentz group metric. The above algebra is the core of the nonlinear realization and, in some sense, of the Invariance Induced Gravity.

\section{GROUP ACTIONS AND BUNDLE MORPHISMS}

Let us now introduce the main ingredients required to specify the structure of the fiber bundle, namely the canonical projection, the sections, etc. We follow the prescription in [39] for constructing the composite fiber bundle, but implement the program for the conformal-affine group.

The composite bundle $\mathbb{P}(\Sigma, H ; \tilde{\pi})$ is comprised of $H$-fibers, base space $\Sigma(M, \mathcal{M})$ and a composite map

$$
\widetilde{\pi} \stackrel{\text { def }}{=} \widetilde{\pi}_{\Sigma M} \circ \prod_{\mathbb{P} \Sigma}: \mathbb{P} \rightarrow \Sigma \rightarrow M
$$

with component projections

$$
\Pi_{\mathbb{P} \Sigma}: \mathbb{P} \rightarrow \Sigma, \tilde{\pi}_{\Sigma M}: \Sigma \rightarrow M
$$

The projection $\Pi_{\mathbb{P} \Sigma}$ maps the point $\left(p \in \mathbb{P}, R_{h} p \in \mathbb{P}\right)$ into point $(x, \xi) \in \Sigma$. There is a correspondence between sections $s_{M \Sigma}: M \rightarrow \Sigma$ and the projection $\Pi_{\mathbb{P} \Sigma}: \mathbb{P} \rightarrow \Sigma$ in the sense that both maps project their functional argument onto elements of $\Sigma$. This is formalized by the relation, $\Pi_{\mathbb{P} \Sigma}(p)=s_{M \Sigma} \circ \pi_{\mathbb{P} M}(p)$. Hence, the total projection is given by

$$
\widetilde{\pi}:=\pi_{\mathbb{P} M}=\tilde{\pi}_{\Sigma M} \circ \Pi_{\mathbb{P} \Sigma}
$$


Associated with the projections $\widetilde{\pi}_{\Sigma M}$ and $\Pi_{\mathbb{P} \Sigma}$ are the corresponding local sections

$$
s_{M \Sigma}: \mathcal{U} \rightarrow \widetilde{\pi}_{\Sigma M}^{-1}(\mathcal{U}) \subset \Sigma, s_{\Sigma \mathbb{P}}: \mathcal{V} \rightarrow \Pi_{\mathbb{P} \Sigma}^{-1}(\mathcal{V}) \subset \mathbb{P},
$$

with neighborhoods $\mathcal{U} \subset M$ and $\mathcal{V} \subset \Sigma$ satisfying

$$
\tilde{\pi}_{\Sigma M} \circ s_{M \Sigma}=(i d)_{M}, \Pi_{\mathbb{P} \Sigma} \circ s_{\Sigma \mathbb{P}}=(i d)_{\Sigma} .
$$

The bundle injection $\widetilde{\pi}^{-1}(\mathcal{U})$ is the inverse image of $\widetilde{\pi}(\mathcal{U})$ and is called the fiber over $\mathcal{U}$. The equivalence class $R_{h} p=p H \in \widetilde{\pi}_{\Sigma M}^{-1}(\mathcal{U})$ of left cosets is the fiber of $\mathbb{P}(\Sigma, H)$ while each orbit $p H$ through $p \in \mathbb{P}$ projects into a single element $Q \in \Sigma$. In analogy to the total bundle projection (37), a total section of $\mathbb{P}$ is given by the total section composition

$$
s_{M \mathbb{P}}=s_{\Sigma \mathbb{P}} \circ s_{M \Sigma} .
$$

Let elements of $G / H$ be labeled by the parameter $\xi$. Functions on $G / H$ are represented by continuous coset functions $c(\xi)$ parameterized by $\xi$. These elements are referred to as cosets to the right of $H$ with respect to $g \in G$. Indeed, the orbits of the right action of $H$ on $G$ are the left cosets $R_{h} g=g H$. For a given section $s_{M \mathbb{P}}(x \in M) \in \pi_{\mathbb{P} M}^{-1}$ with local coordinates $(x, g)$ one can perform decompositions of the partial fibers $s_{M \Sigma}$ and $s_{\Sigma \mathbb{P}}$ as:

$$
\begin{gathered}
s_{M \Sigma}(x)=\widetilde{c}_{M \Sigma}(x) \cdot c=R_{c^{\prime}} \circ \widetilde{c}_{M \Sigma}(x) ; c=c(\xi), \\
s_{\Sigma \mathbb{P}}(x, \xi)=\widetilde{c}_{\Sigma \mathbb{P}}(x, \xi) \cdot a^{\prime}=R_{a^{\prime}} \circ \widetilde{c}_{\Sigma \mathbb{P}}(x, \xi) ; a^{\prime} \in H,
\end{gathered}
$$

with the null sections $\left\{\widetilde{c}_{M \Sigma}(x)\right\}$ and $\left\{\widetilde{c}_{\Sigma \mathbb{P}}(x, \xi)\right\}$ having coordinates $\left(x,(i d)_{\mathcal{M}}\right)$ and $\left(x, \xi,(i d)_{H}\right)$ respectively. A null or zero section is a map that sends every point $x \in M$ to the origin of the fiber $\pi^{-1}(x)$ over $x$, i.e. $\chi_{i}^{-1}(\widetilde{c}(x))=(x, 0)$ in any trivialization. The trivialization map $\chi_{i}^{-1}$ is defined in (9) The identity map appearing in the above trivializations are defined as $(i d)_{\mathcal{M}}: \mathcal{M} \rightarrow \mathcal{M}$ and $(i d)_{H}: H \rightarrow H$. We assume the total null bundle section be given by the composition law

$$
\widetilde{c}_{M \mathbb{P}}=\widetilde{c}_{\Sigma \mathbb{P}} \circ \widetilde{c}_{M \Sigma}
$$

The images of two sections $s_{\Sigma \mathbb{P}}$ and $s_{M \Sigma}$ over $x \in M$ must coincide, implying $s_{\Sigma \mathbb{P}}(x, \xi)=s_{M \Sigma}(x)$. Using (41) with (42), (43) and (44), we arrive at the total bundle section decomposition

$$
s_{M \mathbb{P}}(x)=\widetilde{c}_{M \mathbb{P}}(x) \cdot g=R_{g} \circ \widetilde{c}_{M \mathbb{P}}(x)
$$

provided $g=c \cdot a$ and

$$
\widetilde{c}_{\Sigma \mathbb{P}}=R_{c^{-1}} \circ \widetilde{c}_{\Sigma \mathbb{P}}(x, \xi) \circ R_{c}
$$

The pullback of $\widetilde{c}_{\Sigma \mathbb{P}}$, defined [39] as

$$
\widetilde{c}_{\xi}(x)=\left(s_{M \Sigma}^{*} \widetilde{c}_{\Sigma \mathbb{P}}\right)(x)=\widetilde{c}_{\Sigma \mathbb{P}} \circ s_{M \Sigma}=\widetilde{c}_{\Sigma \mathbb{P}}(x, \xi),
$$

ensures the coincidence of images of sections $\widetilde{c}_{\xi}(x): M \rightarrow \mathbb{P}$ and $\widetilde{c}_{\Sigma \mathbb{P}}(x, \xi): \Sigma \rightarrow \mathbb{P}$, respectively. With the aid of the above results, we arrive at the equation

$$
\widetilde{c}_{\Sigma \mathbb{P}}(x, \xi)=\widetilde{c}_{M \mathbb{P}}(x) \cdot c(\xi),
$$

which will be extremely useful in the following.

\section{NONLINEAR REALIZATIONS AND GENERALIZED GAUGE TRANSFORMATIONS}

The generalized gauge transformation law is obtained by comparing bundle elements $p \in \mathbb{P}$ that differ by the left action of elements of the principal group $G, L_{g \in G}$. An arbitrary element $p \in \mathbb{P}$ can be written in terms of the null section with the aid of (45), (46) and (48) as

$$
p=s_{M \mathbb{P}}(x)=R_{a} \circ \widetilde{c}_{\Sigma \mathbb{P}}(x, \xi), a \in H .
$$


Performing a gauge transformation on $p$, we obtain the orbit $\lambda(p)$ defining a curve through $(x, \xi)$ in $\Sigma$

$$
\lambda(p)=L_{g(x)} \circ p=R_{a^{\prime}} \circ \widetilde{c}_{\Sigma \mathbb{P}}\left(x, \xi^{\prime}\right) ; g(x) \in G, a^{\prime} \in H .
$$

Comparison of (49) with (50) leads to

$$
L_{g(x)} \circ R_{a} \circ \widetilde{c}_{\Sigma \mathbb{P}}(x, \xi)=R_{a^{\prime}} \circ \widetilde{c}_{\Sigma \mathbb{P}}\left(x, \xi^{\prime}\right) .
$$

By virtue of the commutability [44] of left and right group translations of elements belonging to $G$, i.e. $L_{g} \circ R_{h}=$ $R_{h} \circ L_{g}$, Eq.(51) may be recast as

$$
L_{g(x)} \circ \widetilde{c}_{\Sigma \mathbb{P}}(x, \xi)=R_{h} \circ \widetilde{c}_{\Sigma \mathbb{P}}\left(x, \xi^{\prime}\right) .
$$

where $R_{a^{-1}} \circ R_{a^{\prime}} \equiv R_{a^{\prime} a^{-1}}:=R_{h}$ and $a^{\prime} a^{-1} \equiv h \in H$. Equation (52) constitute a generalized gauge transformation. Performing the pullback of (52) with respect to the section $s_{M \Sigma}$ leads to

$$
L_{g(x)} \circ \widetilde{c}_{\xi}(x)=R_{h(\xi, g(x))} \circ \widetilde{c}_{\xi^{\prime}}(x) .
$$

Thus, the left action $L_{g}$ of $G$ is a map that acts on $\mathbb{P}$ and $\Sigma$. In particular, $L_{g}$ acting on fibers defined as orbits of the right action describes diffeomorphisms that transforming fibers over $\widetilde{c}_{\xi}(x)$ into the fibers $\widetilde{c}_{\xi^{\prime}}(x)$ of $\Sigma$ while simultaneously being displaced along $H$ fibers via the action of $R_{h}$. Equation (53) states that nonlinear realizations of $G \bmod H$ is determined by the action of an arbitrary element $g \in G$ on the quotient space $G / H$ transforming one coset into another as

$$
L_{g}: G / H \rightarrow G / H, c(\xi) \rightarrow c\left(\xi^{\prime}\right)
$$

inducing a diffeomorphism $\xi \rightarrow \xi^{\prime}$ on $G / H$. To simplify the action induced by (53) for calculation purposes we proceed as follows. Departing from (47) and substituting $s_{M \Sigma}=R_{c} \circ \widetilde{c}_{M \mathbb{P}}$ we get

$$
\widetilde{c}_{\xi}(x)=\widetilde{c}_{\Sigma \mathbb{P}} \circ R_{c} \circ \widetilde{c}_{M \Sigma}
$$

Using $\widetilde{c}_{M \mathbb{P}} \circ R_{c}=R_{c} \circ \widetilde{c}_{M \mathbb{P}}$, (55) becomes $\widetilde{c}_{\xi}(x)=R_{c} \circ \widetilde{c}_{\Sigma \mathbb{P}} \circ \widetilde{c}_{M \Sigma}=R_{c} \circ \widetilde{c}_{M \mathbb{P}}$, where the last equality follows from use of $\widetilde{c}_{M \mathbb{P}}=\widetilde{c}_{\Sigma \mathbb{P}} \circ \widetilde{c}_{M \Sigma}$. By way of analogy, we assume $\widetilde{c}_{\xi^{\prime}}(x) \equiv R_{c^{\prime}} \circ \widetilde{c}_{M \mathbb{P}}$. Upon substitution of $\widetilde{c}_{\xi^{\prime}}$ into (53) we obtain

$$
L_{g} \circ R_{c} \circ \widetilde{c}_{M \mathbb{P}}=R_{h(\xi, g(x))} \circ R_{c^{\prime}} \circ \widetilde{c}_{M \mathbb{P}}
$$

which after implementing the group actions is equivalent to,

$$
g \cdot \widetilde{c}_{M \mathbb{P}} \cdot c=\widetilde{c}_{M \mathbb{P}} \cdot c^{\prime} \cdot h .
$$

Operating on (57) from the left by $\widetilde{c}_{M \mathbb{P}}^{-1}$ and making use of $g=\widetilde{c}_{M \mathbb{P}}^{-1} g \widetilde{c}_{M \mathbb{P}}$, we get $\left(\widetilde{c}_{M \mathbb{P}}^{-1} \cdot g \cdot \widetilde{c}_{M \mathbb{P}}\right) \cdot c=c^{\prime} \cdot h$ which leads to $g \cdot c_{\xi}=c_{\xi^{\prime}} \cdot h$, or

$$
c^{\prime}=g \cdot c \cdot h^{-1}
$$

in short, where $c \equiv c_{\xi}$ and $c^{\prime} \equiv c_{\xi^{\prime}}$. Observe that the element $h$ is a function whose argument is the couple $(\xi, g(x))$. The transformation rule (58) is in fact the key equation to determine the nonlinear realizations of $G$ and specifies a unique $H$-valued field $h(\xi, g(x))$ on $G / H$.

Consider a family of sections $\{\widehat{c}(x, \xi)\}$ defined [41] on $\Sigma$ by

$$
\widehat{c}(x, \xi):=c \circ \widetilde{c}(x, \xi)=c(\widetilde{c}(x, \xi)) .
$$

Taking $\Pi_{\mathbb{P} \Sigma} \circ R_{h} \circ \widetilde{c}_{\Sigma \mathbb{P}}=\Pi_{\mathbb{P} \Sigma} \circ \widetilde{c}_{\Sigma \mathbb{P}}=(i d)_{\Sigma}$ into account, we can explicitly exhibit the fact that the left action $L_{g}$ of $G$ on the null sections $\widetilde{c}_{\Sigma \mathbb{P}}: \mathbb{P} \rightarrow \Sigma$ induces an equivalence relation between differing elements $\widetilde{c}_{\xi}, \widetilde{c}_{\xi^{\prime}} \in \Sigma$ given by

$$
\Pi_{\mathbb{P} \Sigma} \circ L_{g} \circ \widehat{c}_{\xi}=\Pi_{\mathbb{P} \Sigma} \circ R_{h(\xi, g(x))} \circ \widehat{c}_{\xi^{\prime}}=R_{h(\xi, g(x))} \circ \widetilde{c}_{\xi^{\prime}},
$$

so that

$$
\widetilde{c}_{\xi}:=R_{h(\xi, g(x))} \circ \widetilde{c}_{\xi^{\prime}}=L_{g} \circ \widetilde{c}_{\xi} .
$$

From (61) we can write

$$
\widetilde{c}_{\xi} \stackrel{L_{g}}{\longmapsto} \widetilde{c}_{\xi}=R_{h(\xi, g(x))} \circ \widetilde{c}_{\xi^{\prime}} \forall h \in H .
$$


Equation (62) gives rise to a complete partition of $G / H$ into equivalence classes $\Pi_{\mathbb{P} \Sigma}^{-1}(\xi)$ of left cosets 41 , 45]

$$
c H=\left\{R_{h(\xi, g(x))} \circ c / c \in G / H, \forall h \in H\right\}=\left\{c h_{1}, c h_{2}, \ldots, c h_{n}\right\},
$$

where $c \in(G-H)$ plays the role of the fibers attached to each point of $\Sigma$. The elements $c h_{i}$ are single representatives of each equivalence class $R_{h(\xi, g(x))} \circ c=c H \in \widetilde{\pi}_{\Sigma M}^{-1}(\mathcal{U})$. Thus, any diffeomorphism $L_{g} \circ \widetilde{c}_{\xi}$ on $\Sigma$ together with the $H$-valued function $h(\xi, g(x))$ determine a unique gauge transformation $\widetilde{c}_{\xi}^{\prime}=R_{h(\xi, g(x))} \circ \widetilde{c}_{\xi^{\prime}}$. This demonstrates that gauge transformations are those diffeomorphisms on $\Sigma$ that map fibers over $c(\xi)$ into fibers over $c\left(\xi^{\prime}\right)$ and simultaneously preserve the action of $H$.

\section{THE COVARIANT COSET FIELD TRANSFORMATIONS}

We now proceed to determine the transformation behavior of parameters belonging to $G / H$. The elements of the conformal-affine and Lorentz groups are respectively parameterized about the identity element as

$$
g=e^{i \epsilon^{\alpha} \mathbf{P}_{\alpha}} e^{i \alpha^{\mu \nu}{ }^{\dagger} \mathbf{S}_{\mu \nu}} e^{i \beta^{\mu \nu} \mathbf{L}_{\mu \nu}} e^{i b^{\alpha} \boldsymbol{\Delta}_{\alpha}} e^{i \varphi \mathbf{D}}, h=e^{i u^{\mu \nu} \mathbf{L}_{\mu \nu}} .
$$

Elements of the coset space $G / H$ are coordinatized by

$$
c=e^{-i \xi^{\alpha} \mathbf{P}_{\alpha}} e^{i h^{\mu \nu}{ }^{\dagger} \mathbf{S}_{\mu \nu}} e^{i \zeta^{\alpha} \boldsymbol{\Delta}_{\alpha}} e^{i \phi \mathbf{D}} .
$$

We consider transformations with infinitesimal group parameters $\epsilon^{\alpha}, \alpha^{\mu \nu}, \beta^{\mu \nu}, b^{\alpha}$ and $\varphi$. The transformed coset parameters read $\xi^{\prime \alpha}=\xi^{\alpha}+\delta \xi^{\alpha}, h^{\prime \mu \nu}=h^{\mu \nu}+\delta h^{\mu \nu}, \zeta^{\prime \alpha}=\zeta^{\alpha}+\delta \zeta^{\alpha}$ and $\phi^{\prime}=\phi+\delta \phi$. Note that $u^{\mu \nu}$ is infinitesimal. The translational coset field variations reads

$$
\delta \xi^{\alpha}=-\left(\alpha_{\beta}{ }^{\alpha}+\beta_{\beta}{ }^{\alpha}\right) \xi^{\beta}-\epsilon^{\alpha}-\varphi \xi^{\alpha}-\left[|\xi|^{2} b^{\alpha}-2(b \cdot \xi) \xi^{\alpha}\right] .
$$

For the dilatons we get,

$$
\delta \phi=\varphi+2(b \cdot \xi)-\left\{u_{\beta}^{\alpha} \xi^{\beta}+\epsilon^{\alpha}+\varphi \xi^{\alpha}+\left[b^{\alpha}|\xi|^{2}-2(b \cdot \xi) \xi^{\alpha}\right]\right\} \partial_{\alpha} \phi
$$

Similarly for the special conformal 4-boosts we find,

$$
\begin{aligned}
\delta \zeta^{\alpha}= & u_{\beta}^{\alpha} \zeta^{\beta}+b^{\alpha}-\varphi \zeta^{\alpha}+2\left[(b \cdot \xi) \zeta^{\alpha}-(b \cdot \zeta) \xi^{\alpha}\right]+ \\
& -\left\{u_{\lambda}^{\beta} \xi^{\lambda}+\epsilon^{\beta}+\varphi \xi^{\beta}+\left[b^{\beta}|\xi|^{2}-2(b \cdot \xi) \xi^{\beta}\right]\right\} \partial_{\beta} \zeta^{\alpha} .
\end{aligned}
$$

Observe the homogeneous part of the special conformal coset parameter $\zeta^{\alpha}$ has the same structure as that of the translational parameter $\xi^{\alpha}$ (with the substitutions: $\zeta^{\alpha} \rightarrow-\xi^{\alpha}$ and $-\epsilon^{\alpha} \rightarrow b^{\alpha}$ ). For the shear parameters we obtain

$$
\delta r^{\alpha \beta}=\left(\alpha^{\gamma \alpha}+\beta^{\gamma \alpha}\right) r_{\gamma}{ }^{\beta}+u_{\gamma}^{\beta} r^{\alpha \gamma}+2 b^{[\alpha} \xi^{\rho]} r_{\rho}{ }^{\beta},
$$

where $r^{\alpha \beta}:=e^{h^{\alpha \beta}}$. From $\delta r^{\alpha \beta}$ we obtain the nonlinear Lorentz transformation

$$
u^{\alpha \beta}=\beta^{\alpha \beta}+2 b^{[\alpha} \xi^{\beta]}-\alpha^{\mu \nu} \tanh \left\{\frac{1}{2} \ln \left[r_{\mu}^{\alpha}\left(r^{-1}\right)_{\nu}^{\beta}\right]\right\} .
$$

In the limit of vanishing special conformal 4-boost, this result coincides with that of Pinto et al. [36]. For vanishing shear, the result of Julve et al [37] is obtained.

In this section, all covariant coset field transformations have been determined directly from the nonlinear transformation law (58). We observe that the translational coset parameter transforms as a coordinate under the action of $G$. From the shear coset variation, the explicit form of the nonlinear Lorentz-like transformation was obtained. From (70) it is clear that $u^{\alpha \beta}$ contains the linear Lorentz parameter in addition to conformal and shear contributions via the nonlinear 4-boosts and symmetric $G L_{4}$ parameters. 


\section{THE DECOMPOSITION OF CONNECTIONS IN $\pi_{\mathbb{P} M}: \mathbb{P} \rightarrow M$ INTO COMPONENTS IN $\pi_{\mathbb{P} \Sigma}: \mathbb{P} \rightarrow \Sigma$ AND IN $\pi_{\Sigma M}: \Sigma \rightarrow M$}

At this point, it is useful to classify all possible decompositions of connections in order to achieve all the conformalaffine nonlinear gauge potentials. This step is essential to have all the ingredients to construct the induced metric and dynamics related to the conformal-affine group.

Depending on which bundle is considered, either the total bundle $\mathbb{P} \rightarrow M$ or the intermediate bundles $\mathbb{P} \rightarrow \Sigma$, $\Sigma \rightarrow M$, we may construct corresponding Ehresmann connections for the respective space. With respect to $M$, we have the connection form

$$
\omega=\widetilde{g}^{-1}\left(d+\pi_{\mathbb{P} M}^{*} \Omega_{M}\right) \widetilde{g} .
$$

The gauge potential $\Omega_{M}$ is defined in the standard manner as the pullback of the connection $\omega$ by the null section $\widetilde{c}_{M \mathbb{P}}, \Omega_{M}=\widetilde{c}_{M \mathbb{P}}^{*} \omega \in T^{*}(M)$. With regard to the space $\Sigma$ an alternative form of the connection is given by

$$
\omega=a^{-1}\left(d+\pi_{\mathbb{P} \Sigma}^{*} \Gamma_{\Sigma}\right) a,
$$

where the connection on $\Sigma$ reads $\Gamma_{\Sigma}=\widetilde{c}_{\Sigma p}^{*} \omega$. Carrying out a similar analysis and evaluating the tangent vector $X \in T_{p}(\Sigma)$ at each point $\xi$ along the curve $c_{\xi}$ on the coset space $G / H$ that coincides with the section $\widetilde{c}_{\Sigma \mathbb{P}}^{*}$, we find the gauge transformation law

$$
\omega \rightarrow \omega^{\prime}=a d_{h^{-1}}(d+\omega) .
$$

Comparison of (71) and 72 leads to $\pi_{\mathbb{P} \Sigma}^{*} \Gamma_{\Sigma}=c^{-1}\left(d+\pi_{\mathbb{P} M}^{*} \Omega_{M}\right) c$. Taking account of $\widetilde{c}_{\Sigma \mathbb{P}}^{*} \Pi_{\mathbb{P} \Sigma}^{*}=(i d)_{T^{*}(\Sigma)}$ which follows from $\Pi_{\mathbb{P} \Sigma} \circ \widetilde{c}_{\Sigma \mathbb{P}}=(i d)_{\Sigma}$, we deduce

$$
\Gamma_{\Sigma}=\widetilde{c}_{\Sigma \mathbb{P}}^{*}\left[c^{-1}\left(d+\pi_{\mathbb{P} M}^{*} \Omega_{M}\right) c\right] .
$$

By use of the family of sections pulled back to $\Sigma$ introduced in (59) we find $\widetilde{c}_{\Sigma \mathbb{P}}^{*}\left(c^{-1} d c\right)=\widehat{c}{ }^{-1} d \widehat{c}$ and $\widetilde{c}_{\Sigma \mathbb{P}}^{*} R_{c}^{*}=R_{\widehat{c}}^{*} \widetilde{c}_{\Sigma \mathbb{P}}^{*}$. Recalling $\widetilde{\pi}_{\mathbb{P} M}^{*}=\widetilde{\pi}_{\mathbb{P} \Sigma}^{*} \widetilde{\pi}_{\Sigma M}^{*}$, we get $c^{-1} \widetilde{\pi}_{\mathbb{P} M}^{*} \Omega_{M} c=R_{c}^{*} \widetilde{\pi}_{\mathbb{P} M}^{*} \Omega_{M}$. With these results in hand, we obtain the alternative form of the connection $\Gamma_{\Sigma}$,

$$
\Gamma_{\Sigma}=\widehat{c}^{-1}\left(d+\pi_{\Sigma M}^{*} \Omega_{M}\right) \widehat{c} .
$$

Completing the pullback of $\Gamma_{\Sigma}$ to $M$ by means of $\widetilde{c}_{M \Sigma}$ we obtain, $\Gamma_{M}=\widetilde{c}_{M \Sigma}^{*} \Gamma_{\Sigma}$. By use of $\Gamma_{\Sigma}=\widetilde{c}_{\Sigma \mathbb{P}}^{*} \omega$ and (47) we find $\Gamma_{M}=s_{M \Sigma}^{*} \widetilde{c}_{\Sigma \mathbb{p}}^{*} \omega=\widetilde{c}_{\xi}^{*} \omega$. In terms of the substitution $\widehat{c}(x, \xi) \rightarrow \bar{c}(x)$ where $\bar{c}(x)$ is the pullback of $\widehat{c}(x, \xi)$ to $M$ defined as $\bar{c}(x)=s_{M \Sigma}^{*} \widehat{c}=c\left(\widetilde{c}_{\xi}(x)\right)$, we arrive at the desired result

$$
\Gamma \equiv \Gamma_{M}=\bar{c}^{-1}\left(d+\Omega_{M}\right) \bar{c}
$$

which explicitly relates the connection $\Gamma$ on $\Sigma$ pulled back to $M$ to its counterpart $\Omega_{M}$.

The gauge transformation behavior of $\Gamma$ may be determined directly by use of (29) and the transformation $\widetilde{c}^{\prime}=$ $g \widetilde{c} h^{-1}$. We calculate

$$
\Gamma^{\prime}=h \widetilde{c}^{-1} g^{-1} d\left(g \widetilde{c} h^{-1}\right)+h \widetilde{c}^{-1} \Omega \widetilde{c} h^{-1}+h \widetilde{c}^{-1}\left(d g^{-1}\right) g \widetilde{c} h^{-1}
$$

Observing however, that

$$
h \widetilde{c}^{-1} g^{-1} d\left(g \widetilde{c} h^{-1}\right)=h \widetilde{c}^{-1}\left(g^{-1} d g\right) \widetilde{c} h^{-1}+h \widetilde{c}^{-1} d \widetilde{c} h^{-1}+h d h^{-1},
$$

we obtain

$$
\boldsymbol{\Gamma}^{\prime}=h\left[\widetilde{c}^{-1}(d+\Omega) \widetilde{c}\right] h^{-1}+h d h^{-1}+h \widetilde{c}^{-1} d\left(g g^{-1}\right) \widetilde{c} h^{-1} .
$$

Thus, we arrive at the gauge transformation law

$$
\boldsymbol{\Gamma}^{\prime}=h \boldsymbol{\Gamma} h^{-1}+h d h^{-1} .
$$

According to the Lie algebra decomposition of $\mathfrak{g}$ into $\mathfrak{h}$ and $\mathfrak{c}$, the connection $\Gamma_{\Sigma}$ can be divided into $\boldsymbol{\Gamma}_{H}$ defined on the subgroup $H$ and $\boldsymbol{\Gamma}_{G / H}$ defined on $G / H$. From the transformation law (80) it is clear that $\boldsymbol{\Gamma}_{H}$ transforms inhomogeneously

$$
\boldsymbol{\Gamma}_{H}^{\prime}=h \boldsymbol{\Gamma}_{H} h^{-1}+h d h^{-1},
$$


while $\Gamma_{G / H}$ transforms as a tensor

$$
\boldsymbol{\Gamma}_{G / H}^{\prime}=h \boldsymbol{\Gamma}_{G / H} h^{-1} .
$$

In this regard, only $\Gamma_{H}$ transforms as a true connection. We use the gauge potential $\boldsymbol{\Gamma}$ to define the gauge covariant derivative

$$
\nabla:=(d+\rho(\boldsymbol{\Gamma}))
$$

acting on $\psi$ as $\nabla \psi=(d+\rho(\Gamma)) \psi$ with the desired transformation property

$$
(\nabla \psi(c(\xi)))^{\prime}=\rho(h(\xi, g)) \nabla \psi(c(\xi)) \simeq(1+i u(\xi, g) \rho(H)) \nabla \psi(c(\xi))
$$

leading to

$$
\delta(\nabla \psi(c(\xi)))=i u(\xi, g) \rho(H) \nabla \psi(c(\xi)) .
$$

Let us now classify the conformal-affine gauge potentials considering the various components of the decomposition.

\section{A. Conformal-Affine Nonlinear Gauge Potential in $\pi_{\mathbb{P} M}: \mathbb{P} \rightarrow M$}

The ordinary gauge potential defined on the total base space $M$ reads

$$
\Omega=-i\left(\stackrel{\mathrm{T}}{\Gamma}{ }^{\alpha} \mathbf{P}_{\alpha}+\stackrel{\mathrm{C}}{\Gamma}{ }^{\alpha} \boldsymbol{\Delta}_{\alpha}+\stackrel{\mathrm{D}}{\Gamma} \mathbf{D}+\stackrel{\mathrm{GL}}{\Gamma} \alpha \beta \dagger \boldsymbol{\Lambda}_{\alpha \beta}\right) .
$$

The horizontal basis vectors that span the horizontal tangent space $\mathbb{H}(\mathbb{P})$ of $\pi_{\mathbb{P} M}: \mathbb{P} \rightarrow M$ are given by

$$
E_{i}=\widetilde{c}_{M \mathbb{P} *} \partial_{i}-\Omega_{i} .
$$

The explicit form of the connections (86) are given by

$$
\omega=-i\left[V_{M}^{\mu} \widetilde{\chi}_{\mu}^{\nu} \mathbf{P}_{\nu}-i\left(i \bar{\Theta}_{\left({ }^{\dagger} \boldsymbol{\Lambda}\right)}^{\alpha \beta}+\widetilde{\pi}_{\mathbb{P} M}^{*} \stackrel{\mathrm{GL}}{\Gamma} \alpha \beta\right) \widetilde{\chi}_{\alpha}^{\nu} \widetilde{\chi}_{\beta}^{\nu \dagger} \boldsymbol{\Lambda}_{\mu \nu}+\vartheta_{M}^{\mu} \widetilde{\beta}_{\mu}^{\nu} \boldsymbol{\Delta}_{\nu}-i \widetilde{\pi}_{\mathbb{P} M}^{*} \Phi_{M} \mathbf{D}\right]
$$

where $\bar{\Theta}_{\left({ }^{\dagger} \Lambda\right)}^{\alpha \beta}=\bar{\Theta}_{(\mathbf{L})}^{\alpha \beta}+\bar{\Theta}_{(\mathrm{SY})}^{\alpha \beta}$, with right invariant Maurer-Cartan forms

$$
\bar{\Theta}_{(\mathbf{L})}^{\mu \nu}=i \widetilde{\beta}_{\gamma}^{[\nu \mid} d \widetilde{\beta}^{\mid \mu] \gamma}-2 i d b^{\mu} \epsilon^{\nu} \text { and } \bar{\Theta}_{(\mathrm{SY})}^{\mu \nu}=i \widetilde{\alpha}^{(\nu \mid}{ }_{\gamma} d \widetilde{\alpha}^{\mid \mu) \gamma} .
$$

The linear connection $\Omega_{M}$ varies under the action of $G$ as

$$
\delta \Omega=\Omega^{\prime}-\Omega=\delta \stackrel{\mathrm{T}}{\Gamma}{ }^{\mu} \mathbf{P}_{\mu}+\delta \stackrel{\mathrm{C}}{\Gamma}{ }^{\mu} \boldsymbol{\Delta}_{\mu}+\delta \stackrel{\mathrm{D}}{\Gamma} \mathbf{D}+\delta \stackrel{\mathrm{GL}}{\Gamma} \beta \nu \dagger{ }^{\dagger} \boldsymbol{\Lambda}_{\beta \nu}
$$

where

$$
\begin{gathered}
\delta \stackrel{\mathrm{T}}{\Gamma}^{\mu}=\stackrel{\mathrm{GL}}{\mathrm{D}} \epsilon^{\mu}-\stackrel{\mathrm{T}}{\Gamma}{ }^{\alpha}\left(\alpha_{\alpha}^{\mu}+\beta_{\alpha}^{\mu}+\varphi \delta_{\alpha}^{\mu}\right)-\stackrel{\mathrm{D}}{\Gamma} \epsilon^{\mu}, \\
\delta \Gamma^{\mathrm{C}} \mu=\stackrel{\mathrm{GL}}{D} b^{\mu}-\stackrel{\mathrm{C}}{\Gamma}{ }^{\alpha}\left(\alpha_{\alpha}^{\mu}+\beta_{\alpha}^{\mu}-\varphi \delta_{\alpha}^{\mu}\right)+\stackrel{\mathrm{D}}{\Gamma} b^{\mu}, \\
\delta \stackrel{\mathrm{GL}}{\Gamma} \alpha \beta=\stackrel{\mathrm{GL}}{D}\left(\alpha^{\alpha \beta}+\beta^{\alpha \beta}\right)+\left(\stackrel{\mathrm{T}}{\Gamma}^{[\alpha} b^{\beta]}+\stackrel{\mathrm{C}}{\Gamma}\left[\alpha \epsilon^{\beta]}\right),\right. \\
\delta \stackrel{\mathrm{D}}{\Gamma}=d \varphi+2\left(\stackrel{\mathrm{C}}{\Gamma}^{\alpha} \epsilon_{\alpha}-\stackrel{\mathrm{T}}{\Gamma}{ }^{\alpha} b_{\alpha}\right) .
\end{gathered}
$$

The components of $\bar{\omega}$ on $M$ are identified as spacetime quantities and are determined from the pullback of the corresponding (quotient space) quantities defined on $\Sigma$ :

$$
V_{M}^{\mu}=s_{M \Sigma}^{*} V_{\Sigma}^{\mu}, \vartheta_{M}^{\mu}=s_{M \Sigma}^{*} \vartheta_{\Sigma}^{\mu}, \Phi_{M}=s_{M \Sigma}^{*} \Phi_{\Sigma} \text { and } \Gamma_{M}^{\mu \nu}=s_{M \Sigma}^{*} \Gamma_{\Sigma}^{\mu \nu} .
$$

In the following, we depart from the alternative form of the connection $\omega=a^{-1}\left(d+\prod_{\mathbb{P} \Sigma}^{*} \Gamma_{\Sigma}\right) a, \forall a \in H$ on $\Sigma$. 


\section{B. Conformal-Affine Nonlinear Gauge Potential in $\pi_{\mathbb{P} \Sigma}: \mathbb{P} \rightarrow \Sigma$}

The components of $\omega$ in $\mathbb{P} \rightarrow \Sigma$ are oriented along the Lie algebra basis of $H$

$$
\stackrel{\mathbf{L}}{\omega}=a^{-1}\left(d+i \tilde{\pi}_{\mathbb{P} \Sigma}^{*} \stackrel{\circ}{\Gamma}{ }^{\alpha \beta} \mathbf{L}_{\alpha \beta}\right) a=-i \stackrel{\mathbf{L}}{\omega}{ }^{\alpha \beta} \mathbf{L}_{\alpha \beta}
$$

where

$$
\stackrel{\mathbf{L}}{\omega} \alpha \beta:=\left(i \bar{\Theta}_{(\mathbf{L})}^{\rho \sigma}+\widetilde{\pi}_{\mathbb{P} \Sigma}^{*} \Gamma_{[\mathbf{L}]}^{\rho \sigma}\right) \widetilde{\beta}_{[\rho}^{\alpha} \widetilde{\beta}_{\sigma]}^{\beta} .
$$

\section{Conformal-Affine Nonlinear Gauge Potential on $\Pi_{\Sigma M}: \Sigma \rightarrow M$}

The components of $\omega$ in $\Pi_{\Sigma M}: \Sigma \rightarrow M$ are oriented [39] along the Lie algebra basis of the quotient space $G / H$ belonging to $\Sigma$

$$
\begin{aligned}
& \stackrel{\mathbf{P}}{\omega}=-i a^{-1}\left(\widetilde{\pi}_{\Sigma M}^{*} V_{\Sigma}^{\nu} \mathbf{P}_{\nu}\right) a=-i \stackrel{\mathbf{P}}{\omega}{ }^{\mu} \mathbf{P}_{\mu}, \\
& \stackrel{\boldsymbol{\omega}}{\omega}=-i a^{-1}\left(\widetilde{\pi}_{\Sigma M}^{*} \vartheta_{\Sigma}^{\nu} \boldsymbol{\Delta}_{\nu}\right) a=-i \omega^{\mu} \boldsymbol{\Delta}_{\mu}, \\
& \stackrel{\mathbf{D}}{\omega}=-i a^{-1}\left(\tilde{\pi}_{\Sigma M}^{*} \Phi_{\Sigma} \mathbf{D}\right) a=-i \omega_{[\mathbf{D}]} \mathbf{D}, \\
& \underset{\omega}{\mathrm{SY}}=-i a^{-1}\left(\tilde{\pi}_{\Sigma M}^{*} \Upsilon^{\alpha \beta} \mathbf{S}_{\alpha \beta}\right) a=-i \stackrel{\mathrm{SY}}{\omega}{ }^{\alpha \beta} \mathbf{S}_{\alpha \beta},
\end{aligned}
$$

where

$$
\begin{aligned}
& \stackrel{\mathbf{P}}{\omega} \mu:=\widetilde{\pi}_{\Sigma M}^{*} V_{\Sigma}^{\nu} \widetilde{\beta}_{\nu}^{\mu}, \stackrel{\Delta}{\omega} \mu:=\widetilde{\pi}_{\Sigma M}^{*} \vartheta_{\Sigma}^{\nu} \widetilde{\beta}_{\nu}^{\mu}, \\
& \omega_{[\mathbf{D}]}:=\widetilde{\pi}_{\Sigma M}^{*} \Phi_{\Sigma}, \stackrel{\mathrm{SY}}{\omega} \alpha \beta:=\widetilde{\pi}_{\mathbb{P} \Sigma}^{*} \Upsilon^{\rho \sigma} \widetilde{\alpha}_{(\rho}^{\alpha} \widetilde{\alpha}_{\sigma)}^{\beta} .
\end{aligned}
$$

By direct computation we obtain

$$
\boldsymbol{\Gamma}_{\Sigma}^{\mathrm{CA}}=-i\left(V_{\Sigma}^{\mu} \mathbf{P}_{\mu}+i \vartheta_{\Sigma}^{\mu} \boldsymbol{\Delta}_{\mu}+\Phi_{\Sigma} \mathbf{D}+\Gamma_{\Sigma}^{\alpha \beta} \boldsymbol{\Lambda}_{\alpha \beta}\right)
$$

The nonlinear translational and special conformal connection coefficients $V_{\Sigma}^{\nu}$ and $\vartheta_{\Sigma}^{\nu}$ read

$$
\begin{aligned}
& V_{\Sigma}^{\beta}=\tilde{\pi}_{\Sigma M}^{*}\left[e^{\phi}\left(v^{\beta}(\xi)+r_{\sigma}^{\alpha} \stackrel{\mathrm{C}}{\Gamma}{ }^{\sigma} \mathfrak{B}_{\alpha}^{\beta}(\xi)\right)\right], \\
& \vartheta_{\Sigma}^{\beta}=\widetilde{\pi}_{\Sigma M}^{*}\left[e^{-\phi}\left(v^{\beta}(\zeta)+v^{\sigma}(\xi) \mathfrak{B}_{\sigma}^{\beta}(\zeta)\right)\right],
\end{aligned}
$$

with

$$
v_{i}^{\beta}(\xi):=r_{\sigma}^{\beta}\left({ }^{\dagger} D_{i} \xi^{\sigma}+\stackrel{\mathrm{D}}{\Gamma}_{i} \xi^{\sigma}+\stackrel{\mathrm{T}}{\Gamma} \underset{i}{\sigma}\right), \mathfrak{B}_{\alpha}^{\rho}(\xi):=\left(|\xi|^{2} \delta_{\alpha}^{\rho}-2 \xi_{\alpha} \xi^{\rho}\right) .
$$

The nonlinear $G L_{4}$ and dilaton connections are given by

$$
\begin{gathered}
\Gamma_{\Sigma}^{\mu \nu}=\widehat{\Gamma}^{\mu \nu}+2 \zeta^{[\mu} \varpi^{\nu]}, \\
\Phi=\widetilde{\pi}_{\Sigma M}^{*}\left(\zeta_{\beta} \varpi^{\beta}\right)-\frac{1}{2} d \phi,
\end{gathered}
$$


with

$$
\widehat{\Gamma}^{\mu \nu}:=\widetilde{\pi}_{\Sigma M}^{*}\left[\left(r^{-1}\right)_{\sigma}^{\mu} \stackrel{\mathrm{GL}}{\Gamma}{ }^{\sigma \beta} r_{\beta}^{\nu}-\left(r^{-1}\right)_{\sigma}^{\mu} d r^{\sigma \nu}\right]
$$

and

$$
\varpi^{\nu}:=v^{\nu}+r_{\alpha}^{\nu} \stackrel{\mathrm{C}}{\Gamma} \alpha
$$

The nonlinear $G L_{4}$ connection can be expanded in the $G L_{4}$ Lie algebra according to $\Gamma^{\alpha \beta} \dagger^{\dagger} \boldsymbol{\Lambda}_{\alpha \beta}=\stackrel{\circ}{\Gamma}{ }^{\alpha \beta} \mathbf{L}_{\alpha \beta}+\Upsilon^{\alpha \beta}$ ${ }^{\dagger} \mathbf{S}_{\alpha \beta}$, where

$$
\stackrel{\circ}{\Gamma} \underset{\Sigma}{\alpha \beta}:=\widehat{\Gamma}^{[\alpha \beta]}+2 \zeta^{[\alpha} \varpi^{\beta]}, \Upsilon_{\Sigma}^{\alpha \beta}:=\widehat{\Gamma}^{(\alpha \beta)} .
$$

The symmetric $G L_{4}$ (shear) gauge fields $\Upsilon$ are distortion fields describing the difference between the general linear connection and the Levi-Civita connection.

We define the (group) algebra bases $e_{\nu}$ and $h_{\nu}$ dual to the translational and special conformal 1-forms $V^{\mu}$ and $\vartheta^{\mu}$ as

$$
\begin{aligned}
& e_{\mu}:=e_{\mu}^{i} s_{M \Sigma *} \partial_{i}=\partial_{\xi^{\mu}}-e_{\mu}^{i} \widetilde{e}_{i}, \\
& h_{\mu}:=h_{\mu}^{i} s_{M \Sigma *} \partial_{i}=\partial_{\zeta^{\mu}}-h_{\mu}{ }_{\mu}^{i} \widetilde{h}_{i},
\end{aligned}
$$

with corresponding tetrad-like components

$$
\begin{aligned}
e_{i}^{\mu}(\xi) & =e^{\phi}\left(v_{i}^{\mu}(\xi)+r_{\sigma}^{\alpha}{ }_{\sigma}^{\mathrm{C}}{ }_{i}{ }^{\sigma} \mathfrak{B}_{\alpha}^{\mu}(\xi)\right), \\
h_{i}^{\mu}(\xi, \zeta) & =e^{-\phi}\left(v_{\rho}^{\mu}(\zeta)+v_{i}^{\sigma}(\xi) \mathfrak{B}_{\sigma}{ }^{\mu}(\zeta)\right),
\end{aligned}
$$

and basis vectors (on $M$ )

$$
\widetilde{e}_{j}(\xi)=\widetilde{c}_{M \Sigma *} \partial_{j}-e^{\phi}\left[r_{\mu}{ }^{\nu}\left(\stackrel{\mathrm{GL}}{\Gamma}{ }_{j \alpha}^{\mu} \xi^{\alpha}+\stackrel{\mathrm{D}}{\Gamma}_{j} \xi^{\mu}+\stackrel{\mathrm{T}}{\Gamma}{ }_{j}^{\mu}\right)+\stackrel{\mathrm{C}}{\Gamma}_{j}{ }_{j} r^{\mu}{ }_{\sigma} \mathfrak{B}_{\mu}^{\nu}(\xi)\right] \partial_{\xi^{\nu}}
$$

and

$$
\widetilde{h}_{j}(\xi, \zeta)=\widetilde{c}_{M \Sigma *} \partial_{j}+e^{-\phi}\left[r_{\rho}^{\mu}\left(\stackrel{\mathrm{GL}}{\Gamma}{ }_{j \alpha}^{\rho} \zeta^{\alpha}+\stackrel{\mathrm{C}}{\Gamma} \underset{j}{\rho}\right)+r_{\sigma}^{\gamma}\left(\stackrel{\mathrm{GL}}{\Gamma}_{j \alpha}^{\sigma} \xi^{\alpha}+\stackrel{\mathrm{D}}{\Gamma}_{j} \xi^{\sigma}+\stackrel{\mathrm{T}}{\Gamma}{ }_{j}^{\sigma}\right) \mathfrak{B}_{\gamma}^{\mu}(\zeta)\right] \partial_{\zeta^{\mu}} .
$$

Here $v^{\beta}(\zeta)=v^{\beta}(\xi \rightarrow \zeta), \mathfrak{B}_{\alpha}^{\beta}(\zeta)=\mathfrak{B}_{\alpha}^{\rho}(\xi \rightarrow \zeta)$. By definition, the basis vectors satisfy the orthogonality relations

$$
\left\langle V_{\Sigma}^{\mu} \mid \widetilde{e}_{j}\right\rangle=0,\left\langle\vartheta_{\Sigma}^{\mu} \mid \widetilde{h}_{j}\right\rangle=0,\left\langle V^{\mu} \mid e_{\nu}\right\rangle=\delta_{\nu}^{\mu},\left\langle\vartheta^{\mu} \mid h_{\nu}\right\rangle=\delta_{\nu}^{\mu} .
$$

We introduce the dilatonic and symmetric $G L_{4}$ algebra bases

$$
b:=\partial_{\phi}-d^{i} \widetilde{d}_{i}, \quad f_{\mu \nu}:=\partial_{\alpha^{\mu \nu}}-f_{\mu \nu}^{i} \widetilde{f}_{i}
$$

with auxiliary soldering components $d_{i}$ and $f_{i}{ }^{\mu \nu}$,

$$
\begin{aligned}
d_{i} & =\zeta_{\sigma} r^{\sigma}{ }_{\rho}\left({ }^{\dagger} D_{i}^{\mathrm{GL}} \xi^{\rho}+\stackrel{\mathrm{D}}{\Gamma}_{i} \xi^{\rho}+\stackrel{\mathrm{T}}{\Gamma}{ }_{i}^{\rho}+\stackrel{\mathrm{C}}{\Gamma}_{i}^{\rho}\right)-\frac{1}{2} \partial_{i} \phi, \\
f_{i}^{\mu \nu} & =\left(r^{-1}\right)_{\sigma}^{\mu}{ }_{\Gamma}^{\mathrm{GL}}{ }_{i}^{\sigma \beta} r_{\beta}^{\nu}-\left(r^{-1}\right)_{\sigma}^{\mu} \partial_{i} r^{\sigma \nu} .
\end{aligned}
$$

The coordinate bases $\widetilde{d}_{j}$ and $\widetilde{f}_{j}$ read

$$
\widetilde{d}_{j}(\xi, \zeta, \phi, h):=\widetilde{c}_{M \Sigma *} \partial_{j}-\zeta_{\sigma} r_{\rho}^{\sigma}\left({ }^{\dagger}{ }^{\mathrm{GL}}{ }_{j \gamma}^{\rho} \xi^{\gamma}+\stackrel{\mathrm{D}}{\Gamma}_{j} \xi^{\rho}+\stackrel{\mathrm{T}}{\Gamma}_{j}^{\rho}+\stackrel{\mathrm{C}}{\Gamma}_{j}^{\rho}\right) \partial_{\phi},
$$


and

$$
\widetilde{f}_{j}(\xi, h):=\widetilde{c}_{M \Sigma *} \partial_{j}-\left(\left(r^{-1}\right){ }_{\sigma}^{(\mu \mid} \stackrel{\mathrm{GL}}{\Gamma}_{j}^{\sigma \beta} r_{\beta}^{\mid \nu)}-\left(r^{-1}\right)_{\sigma}^{(\mu \mid} \partial_{j} r^{\sigma \mid \nu)}\right) \partial_{h^{\mu \nu}}
$$

The bases satisfy

$$
\left\langle\Phi \mid \widetilde{d}_{i}\right\rangle=0,\left\langle\Upsilon^{\alpha \beta} \mid \tilde{f}_{i}\right\rangle=0,\langle\Phi \mid b\rangle=I,\left\langle\Upsilon^{\alpha \beta} \mid f_{\mu \nu}\right\rangle=\delta_{\mu}^{\alpha} \delta_{\nu}^{\beta} .
$$

With the basis vectors and tetrad components in hand, we observe

$$
\begin{gathered}
V_{M}^{\mu}:=d x^{i} \otimes e_{i}^{\mu}, \vartheta_{M}^{\mu}:=d x^{i} \otimes h_{i}^{\mu}, \\
\Phi_{M}:=d x^{i} \otimes e_{i}^{\alpha}\left\langle\Phi \mid e_{\alpha}\right\rangle=d x^{i} \otimes d_{i} .
\end{gathered}
$$

The symmetric and antisymmetric $G L_{4}$ connection pulled back to $M$ is given by

$$
\begin{gathered}
\Upsilon_{M}^{\mu \nu}=d x^{i} \otimes e_{i}^{\alpha}\left\langle\Upsilon_{\Sigma}^{\mu \nu} \mid e_{\alpha}\right\rangle:=d x^{i} \otimes f_{i}^{\mu \nu}, \\
\stackrel{\circ}{\Gamma}_{M}^{\mu \nu}=d x^{i} \otimes e_{i}^{\alpha}\left\langle\stackrel{\circ}{\Gamma}_{\Sigma}^{\mu \nu} \mid e_{\alpha}\right\rangle:=d x^{i} \otimes \stackrel{\circ}{\Gamma}_{i}^{\mu \nu} .
\end{gathered}
$$

With the aid of (123) and (124), we determine

$$
V_{i}^{\beta}:=e_{i}^{\alpha}\left\langle V_{\Sigma}^{\beta} \mid e_{\alpha}\right\rangle=e_{i}^{\alpha} \delta_{\alpha}^{\beta}=e_{i}^{\beta}, \vartheta_{i}^{\beta} \equiv h_{i}^{\beta}, \Upsilon_{i}^{\mu \nu} \equiv f_{i}^{\mu \nu}, \Phi_{i} \equiv d_{i} .
$$

The horizontal tangent subspace vectors in $\widetilde{\pi}_{\mathbb{P} \Sigma}: \mathbb{P} \rightarrow \Sigma$ are given by

$$
\begin{gathered}
\widehat{E}_{i}=\widetilde{c}_{M \mathbb{P} *} \widetilde{e}_{i}+i \widetilde{c}_{M \Sigma *}\left\langle\stackrel{\circ}{\Gamma}^{\alpha \beta} \mid \widetilde{e}_{i}\right\rangle \widehat{\Re}_{\alpha \beta}^{\mathrm{Int}}, \\
\widehat{E}_{\mu}=\widetilde{c}_{\Sigma \mathbb{P} *} \widetilde{e}_{\mu}+i\left\langle\stackrel{\circ}{\Gamma}^{\alpha \beta} \mid \widetilde{e}_{\mu}\right\rangle \widehat{\mathfrak{R}}_{\alpha \beta}^{\mathrm{Int}},
\end{gathered}
$$

and satisfy

$$
\left\langle\stackrel{\mathrm{L}}{\omega} \mid \widehat{E}_{j}\right\rangle=0=\left\langle\mathrm{L} \mid \widehat{E}_{\mu}\right\rangle .
$$

The right invariant fundamental vector operator appearing in (126) or (127) is given by

$$
\widehat{\Re}(\mathbf{L})=i\left(\widetilde{\beta}_{[\mu \mid}^{\gamma} \frac{\partial}{\partial \widetilde{\beta}^{\mid \nu] \gamma}}+\epsilon_{[\mu} \frac{\partial}{\partial \epsilon^{\nu]}}\right) .
$$

On the other hand, the vertical tangent subspace vector in $\widetilde{\pi}_{\mathbb{P} \Sigma}: \mathbb{P} \rightarrow \Sigma$ satisfies

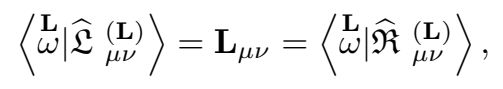

where

$$
\widehat{\mathfrak{L}} \underset{\mu \nu}{(\mathbf{L})}=i \widetilde{\beta}_{\gamma[\mu \mid} \frac{\partial}{\partial \widetilde{\beta}_{\gamma}^{\mid \nu]}}, \widehat{\mathfrak{R}}_{\mu \nu}^{(\mathbf{L})}=i\left(\widetilde{\beta}_{[\mu \mid}^{\gamma} \frac{\partial}{\partial \widetilde{\beta}^{\mid \nu] \gamma}}+\epsilon_{[\mu} \frac{\partial}{\partial \epsilon^{\nu]}}\right)
$$

and $\widetilde{\beta}_{\mu}{ }^{\nu}:=e^{\beta_{\mu}{ }^{\nu}}=\delta_{\mu}{ }^{\nu}+\beta_{\mu}{ }^{\nu}+\frac{1}{2 !} \beta_{\mu}^{\gamma} \beta_{\gamma}^{\nu}+\cdots$. The horizontal tangent subspace vectors in $\Pi_{\Sigma M}: \Sigma \rightarrow M$ are given by

$$
\widetilde{E}_{j}=\widetilde{c}_{\Sigma \mathbb{P} *} \widetilde{e}_{j}, \widetilde{H}_{j}=\widetilde{c}_{\Sigma \mathbb{P} *} \widetilde{h}_{j}, \widehat{E}_{i}^{(\mathbf{D})}=\widetilde{c}_{\Sigma \mathbb{P} *} \widetilde{d}_{j}, \widetilde{E}_{j}=\widetilde{c}_{\Sigma \mathbb{P} *} \widetilde{f}_{j},
$$

and satisfy

$$
\left\langle\stackrel{\mathbf{P}}{\omega} \mid \widetilde{E}_{j}\right\rangle=0,\left\langle\stackrel{\Delta}{\omega} \mid \widetilde{H}_{j}\right\rangle=0,\left\langle\stackrel{\mathrm{SY}}{\omega} \mid \breve{E}_{j}\right\rangle=0,\left\langle{ }_{\omega}^{\mathbf{D}} \mid \widehat{E}_{i}^{(\mathbf{D})}\right\rangle=0
$$


The vertical tangent subspace vectors in $\Pi_{\Sigma M}: \Sigma \rightarrow M$ are given by

$$
\widetilde{E}_{\mu}=\widetilde{c}_{\Sigma \mathbb{P} *} \widehat{\mathfrak{L}}_{\mu}^{(\mathbf{P})}, \breve{E}_{\alpha \beta}=\widetilde{c}_{\Sigma \mathbb{P} *} \widehat{\mathfrak{L}}_{\alpha \beta}^{(\mathrm{SY})}, \widetilde{H}_{\mu}=\widetilde{c}_{\Sigma \mathbb{P} *} \widehat{\mathfrak{L}}_{\mu}^{(\Delta)}, \widehat{E}^{(\mathbf{D})}=\widetilde{c}_{\Sigma \mathbb{P} *} \widehat{\mathfrak{L}}^{(\mathbf{D})},
$$

and satisfy

$$
\left\langle\stackrel{\mathbf{P}}{\omega} \mid \widetilde{E}_{\mu}\right\rangle=\mathbf{P}_{\mu},\left\langle\stackrel{\Delta}{\omega} \mid \widetilde{H}_{\mu}\right\rangle=\mathbf{\Delta}_{\mu},\left\langle\stackrel{\mathrm{SY}}{\omega} \mid \breve{E}_{\alpha \beta}\right\rangle={ }^{\dagger} \mathbf{S}_{\alpha \beta},\left\langle\stackrel{\mathrm{D}}{\omega} \mid \widehat{E}^{(\mathbf{D})}\right\rangle=\mathbf{D} .
$$

The left invariant fundamental vector operators appearing in (134) are readily computed, the result being

$$
\begin{gathered}
\widehat{\mathfrak{L}}_{\mu}^{(\mathbf{P})}=i \widetilde{Q}_{\mu}^{\nu} \frac{\partial}{\partial \epsilon^{\nu}}, \widehat{\mathfrak{L}}_{\mu}^{(\boldsymbol{\Delta})}=i \widetilde{W}_{\mu}^{\nu} \frac{\partial}{\partial b^{\nu}}, \\
\widehat{\mathfrak{L}}_{\alpha \beta}^{(\mathrm{SY})}=i \widetilde{\alpha}_{\gamma(\mu \mid} \frac{\partial}{\partial \widetilde{\alpha}_{\gamma}{ }^{(\nu)}}, \widehat{\mathfrak{L}}(\mathbf{D})=-i \epsilon^{\beta} \frac{\partial}{\partial \epsilon^{\beta}},
\end{gathered}
$$

where $\widetilde{\alpha}_{\mu}^{\nu}:=e^{\alpha_{\mu}^{\nu}}=\alpha_{\mu}^{\nu}+\alpha_{\mu}^{\nu}+\frac{1}{2 !} \alpha_{\mu}^{\gamma} \alpha_{\gamma}^{\nu}+\cdots, \widetilde{Q}_{\sigma}^{\alpha}:=\left(\widetilde{\chi}_{\sigma}^{\alpha}+\delta_{\sigma}^{\alpha} e^{\varphi}\right), \widetilde{W}_{\sigma}^{\alpha}:=\left(\widetilde{\chi}_{\sigma}^{\alpha}+\delta_{\sigma}^{\alpha} e^{-\varphi}\right)$ satisfying $\left(\widetilde{Q}^{-1}\right)_{\sigma}^{\alpha}=\widetilde{Q}_{\sigma}^{\alpha}$ and $\left(\widetilde{W}^{-1}\right)_{\sigma}^{\alpha}=\widetilde{W}_{\sigma}^{\alpha}$. Making use of the transformation law of the nonlinear connection (80) we obtain

$$
\delta \Gamma=\delta V^{\alpha} \mathbf{P}_{\alpha}+\delta \vartheta^{\alpha} \boldsymbol{\Delta}_{\alpha}+2 \delta \Phi \mathbf{D}+\delta \boldsymbol{\Gamma}^{\alpha \beta \dagger} \boldsymbol{\Lambda}_{\alpha \beta}
$$

where

$$
\delta V^{\nu}=u_{\alpha}{ }^{\nu} V^{\alpha}, \delta \vartheta^{\nu}=u_{\alpha}{ }^{\nu} \vartheta^{\alpha}, \delta \Phi=0, \delta \boldsymbol{\Gamma}^{\alpha \beta}={ }^{\dagger} \stackrel{\mathrm{GL}}{\nabla} u^{\alpha \beta}
$$

From $\delta \boldsymbol{\Gamma}^{\alpha \beta}=\dagger^{\mathrm{GL}} \nabla u^{\alpha \beta}$ we observe that

$$
\delta \Gamma^{[\alpha \beta]}=\stackrel{\circ}{\nabla} u^{\alpha \beta}, \delta \Upsilon_{\alpha \beta}=2 u_{(\alpha \mid}^{\rho} \Upsilon_{\rho \mid \beta)} .
$$

According to (138), the nonlinear translational and special conformal gauge fields transform as contravariant vector valued 1-forms under $H$, the antisymmetric part of $\boldsymbol{\Gamma}^{\alpha \beta}$ transforms inhomogeneously as a gauge potential and the nonlinear dilaton gauge field $\Phi$ transforms as a scalar valued 1-form. From (139) it is clear that the symmetric part of $\Gamma^{\alpha \beta}$ is a tensor valued 1-form. Being 4-covectors we identify $V^{\nu}$ as coframe fields. The connection coefficient $\stackrel{\circ}{\Gamma} \alpha \beta$ serves as the gravitational gauge potential. The remaining components of $\boldsymbol{\Gamma}$, namely $\vartheta, \Upsilon$ and $\Phi$ are dynamical fields of the theory. As will be seen in the following Section, the tetrad components of the coframe are used in conjunction with the $H$-metric to induce a spacetime metric on $M$.

At this point, we have discussed all the mathematical tools that we will use to realize the Invariance Induced Gravity. In next Sections, we will proceed with the program of constructing the induced metric, the action functional and the field equations. This will be the original contribution of the present review article where we intend to give a comprehensive approach to gravity derived from group deformations and conformal-affine transformations.

\section{THE INDUCED METRIC}

The bundle structure of gravitation, together with the conformal-affine algebra and the nonlinear realizations of gauge transformations (in particular the classification of gauge potentials), provide us all the tools to realize the Invariance Induced Gravity. In the following part of the paper, we will derive the gravitational field and internal symmetry (spin) quantities showing that they are nothing else but realizations of the local conformal-affine transformations. In other words, the deformations of the Poincaré group give rise to gravity and internal symmetries (see also [29, 40].

Since the Lorentz group $H$ is a subgroup of $G$, we inherit the invariant $\left(\delta o_{\alpha \beta}=\delta o^{\alpha \beta}=0\right)$ (constant) metric of $H$, where $o^{\alpha \beta}=o_{\alpha \beta}=\operatorname{diag}(-,+,+,+)$. With the aid of $o_{\alpha \beta}$ and the tetrad components $e_{i}{ }^{\alpha}$ given in (112), we define the spacetime metric

$$
g_{i j}=e_{i}^{\alpha} e_{j}^{\beta} o_{\alpha \beta}
$$

Observing ${ }^{\dagger}{ }^{\mathrm{GL}} o_{\alpha \beta}=-2 \Upsilon_{\alpha \beta}$ (where we used $d o_{\alpha \beta}=0$ ) and taking account of the (second) transformation property (139), we interpret $\Upsilon_{\alpha \beta}$ as a sort of nonmetricity, i.e. a deformation (or distortion) gauge field that describes the 
difference between the general linear connection and the Levi-Civita connection of Riemannian geometry [40]. In the limit of vanishing gravitational interactions, we have $\stackrel{\mathrm{T}}{\Gamma}{ }^{\sigma} \sim \stackrel{\mathrm{C}}{\Gamma}{ }^{\sigma} \sim \stackrel{\circ}{\Gamma}{ }_{\beta}^{\alpha} \sim \Upsilon^{\alpha}{ }_{\beta} \sim \Phi \rightarrow 0$, $r_{\sigma}^{\beta} \rightarrow \delta_{\sigma}^{\beta}$ (to first order) and GL

${ }^{\dagger} D \xi^{\sigma} \rightarrow d \xi^{\sigma}$. Under these conditions, the coframe reduces to $V^{\beta} \rightarrow e^{\phi} \delta_{\alpha}^{\beta} d \xi^{\alpha}$ leading to the spacetime metric

$$
g_{i j} \rightarrow e^{2 \phi} \delta_{\alpha}^{\rho} \delta_{\beta}^{\sigma}\left(\partial_{i} \xi^{\alpha}\right)\left(\partial_{j} \xi^{\beta}\right) o_{\rho \sigma}=e^{2 \phi}\left(\partial_{i} \xi^{\alpha}\right)\left(\partial_{j} \xi^{\beta}\right) o_{\alpha \beta}
$$

characteristic of a Weyl geometry. In this sense, the invariance properties induce the gravitational field and generalize results in [40]. It is worth noting that conformal transformations of the metric tensor constitute only a part of the whole deformation field.

\section{THE CARTAN STRUCTURE EQUATIONS}

Our task is now to deduce the dynamics. Using the nonlinear gauge potentials derived in Eqs.(103), (105), (106), the covariant derivative defined on $\Sigma$ pulled back to $M$ has the form

$$
\nabla:=d-i V^{\alpha} \mathbf{P}_{\alpha}-i \vartheta^{\alpha} \boldsymbol{\Delta}_{\alpha}-2 i \Phi \mathbf{D}-i \Gamma^{\alpha \beta \dagger} \boldsymbol{\Lambda}_{\alpha \beta}
$$

By using (142) together with the relevant Lie algebra commutators, we obtain the the bundle curvature

$$
\mathbb{F}:=\nabla \wedge \nabla=-i \mathcal{T}^{\alpha} \mathbf{P}_{\alpha}-i \mathcal{K}^{\alpha} \boldsymbol{\Delta}_{\alpha}-i \mathcal{Z} \mathbf{D}-i \mathbb{R}_{\alpha}{ }^{\beta}{ }^{\dagger} \boldsymbol{\Lambda}^{\alpha}{ }_{\beta}
$$

The field strength components of $\mathbb{F}$ are given by the first Cartan structure equations. They are respectively, the projectively deformed, $\Upsilon$-distorted translational field strength

$$
\mathcal{T}^{\alpha}:=\stackrel{\mathrm{GL}}{\nabla} V^{\alpha}+2 \Phi \wedge V^{\alpha}
$$

the projectively deformed, $\Upsilon$-distorted special conformal field strength

$$
\mathcal{K}^{\alpha}:=\stackrel{\mathrm{GL}}{\nabla} \vartheta^{\alpha}-2 \Phi \wedge \vartheta^{\alpha},
$$

the $\Psi$-deformed Weyl homothetic curvature 2-form (dilaton field strength)

$$
\mathcal{Z}:=d \Phi+\Psi, \Psi=V \cdot \vartheta-\vartheta \cdot V
$$

and the general conformal-affine curvature

$$
\mathbb{R}^{\alpha \beta}:=\widehat{R}^{\alpha \beta}+\Psi^{\alpha \beta}
$$

with

$$
\widehat{R}^{\alpha \beta}:=\mathfrak{R}^{\alpha \beta}+\mathcal{R}^{\alpha \beta}, \Psi^{\alpha \beta}:=V^{[\alpha} \wedge \vartheta^{\beta]} .
$$

Operator $\dagger^{\mathrm{GL}} \nabla$ denotes the nonlinear covariant derivative built from volume preserving (VP) connection (i.e. excluding $\Phi)$ forms respectively. The $\Upsilon$ and $\stackrel{\circ}{\Gamma}$-affine curvatures in (148) read

$$
\begin{aligned}
& \mathfrak{R}^{\alpha \beta}:=\stackrel{\circ}{\nabla} \Upsilon^{\alpha \beta}+\Upsilon_{\gamma}^{\alpha} \wedge \Upsilon^{\gamma \beta}, \\
& \mathcal{R}^{\alpha \beta}:=d \stackrel{\circ}{\Gamma}{ }^{\alpha \beta}+\stackrel{\circ}{\Gamma}_{\gamma}^{\alpha} \wedge \stackrel{\circ}{\Gamma} \gamma \beta
\end{aligned}
$$

respectively. Operator $\stackrel{\circ}{\nabla}$ is defined with respect to the restricted connection $\stackrel{\circ}{\Gamma} \alpha \beta$ given in (109).

The field strength components of the bundle curvature have the following group variations

$$
\delta \mathbb{R}_{\alpha}^{\beta}=u_{\alpha}^{\gamma} \mathbb{R}_{\gamma}^{\beta}-u_{\gamma}^{\beta} \mathbb{R}_{\alpha}^{\gamma}, \delta \mathcal{Z}=0, \delta \mathcal{T}^{\alpha}=-u_{\beta}^{\alpha} \mathcal{T}^{\beta}, \delta \mathcal{K}^{\alpha}=-u_{\beta}^{\alpha} \mathcal{K}^{\beta}
$$

A gauge field Lagrangian is built from polynomial combinations of the strength $\mathbb{F}$ defined as

$$
\mathbb{F}(\Gamma(\Omega, D \xi), d \Gamma):=\nabla \wedge \nabla=d \Gamma+\Gamma \wedge \Gamma .
$$

Now we have all the ingredients to derive the conservation laws that constitute a fundamental result of our approach rendering the theory self-consistent. 


\section{THE BIANCHI IDENTITIES}

In what follows, the Bianchi identities play a central role being the conservation laws of the theory. We therefore derive them presently.

1a) The $1^{\text {st }}$ translational Bianchi identity reads,

$$
\stackrel{\mathrm{GL}}{\nabla} \mathcal{T}^{a}=\widehat{R}_{\beta}^{\alpha} \wedge V^{\beta}+\Phi \wedge T^{a}+2 d\left(\Phi \wedge V^{\alpha}\right)
$$

1b) Similarly to the case in (1a), the $1^{\text {st }}$ conformal Bianchi identities are respectively given by

$$
\stackrel{\mathrm{GL}}{\nabla} \mathcal{K}^{a}=\widehat{R}_{\beta}^{\alpha} \wedge \vartheta^{\beta}-\Phi \wedge \mathcal{K}^{a}-2 d\left(\Phi \wedge \vartheta^{\alpha}\right)
$$

2a) The $\Upsilon$ and $\stackrel{\circ}{\Gamma}$-affine component of the $2^{\text {nd }}$ Bianchi identity is given by

$$
{ }^{\mathrm{GL}} \nabla \mathfrak{R}^{\alpha \beta}=2 \mathfrak{R}^{(\alpha \mid}{ }_{\gamma}^{\gamma \mid \beta)},{ }^{\dagger} \stackrel{\mathrm{GL}}{\nabla} \mathcal{R}^{\alpha \beta}=0,
$$

respectively. Hence, the generalized $2^{\text {nd }}$ Bianchi identity is given by

$$
\dagger \stackrel{\mathrm{GL}}{\nabla} \widehat{R}_{\beta}^{\alpha}=2 \mathfrak{R}_{\gamma}^{(\alpha \mid} \Upsilon^{\gamma \mid \rho)} o_{\rho \beta}
$$

Since the full curvature $\mathbb{R}^{\alpha \beta}$ is proportional to $\Psi^{\alpha \beta}$, it is necessary to consider

$$
{ }^{\mathrm{GL}} \nabla \Psi^{\alpha \beta}={ }^{\dagger} \mathcal{T}^{\alpha} \wedge \vartheta^{\beta}+V^{\alpha} \wedge{ }^{\dagger} \mathcal{K}^{\beta}
$$

from which we conclude

$$
{ }^{\dagger} \stackrel{\mathrm{GL}}{\nabla} \mathbb{R}^{\alpha \beta}=2 \mathfrak{R}^{(\alpha \mid}{ }_{\gamma} \Upsilon^{\gamma \mid \beta)}+{ }^{\dagger} \mathcal{T}^{\alpha} \wedge \vartheta^{\beta}+V^{\alpha} \wedge{ }^{\dagger} \mathcal{K}^{\beta}
$$

2c) The dilatonic component of the $2^{\text {nd }}$ Bianchi identity is given by

$$
\stackrel{\mathrm{GL}}{\nabla} \mathcal{Z}=d Z+\stackrel{\mathrm{GL}}{\nabla}(V \wedge \vartheta)=\stackrel{\mathrm{GL}}{\nabla} \Psi+\Phi \wedge \Psi
$$

From the definition of $\Psi$, we obtain

$$
\nabla \Psi=\mathcal{T}^{\alpha} \wedge \vartheta_{\alpha}+V_{\alpha} \wedge \mathcal{K}^{\alpha}+\Phi \wedge\left(V_{\alpha} \wedge \vartheta^{\alpha}\right)
$$

Defining

$$
\Sigma^{\mu \nu}:=\mathbf{B}^{\mu \nu}+\Psi^{\mu \nu}, \mathbf{B}^{\mu \nu}:=B^{\mu \nu}+\mathcal{B}^{\mu \nu}, B^{\mu \nu}:=V^{\mu} \wedge V^{\nu}, \mathcal{B}^{\mu \nu}:=\vartheta^{\mu} \wedge \vartheta^{\nu}
$$

and asserting $V^{\alpha} \wedge \vartheta_{\alpha}=0$, we find $\Sigma_{\mu \nu} \wedge \Sigma^{\mu \nu}=0$. Using this result,we obtain

$$
\nabla \Psi=\mathcal{T}^{\alpha} \wedge \vartheta_{\alpha}+V_{\alpha} \wedge \mathcal{K}^{\alpha}
$$

The last step is now to derive the field equations.

\section{THE ACTION FUNCTIONAL AND THE FIELD EQUATIONS}

We seek an action for a local gauge theory based on the $C A(3,1)$ symmetry group. We consider the $3 D$ topological invariants $\mathbb{Y}$ of the non-Riemannian manifold of conformal-affine connections. Our objective is the $4 D$ boundary terms $\mathbb{B}$ obtained by means of exterior differentiation of these $3 D$ invariants, i.e. $\mathbb{B}=d \mathbb{Y}$. The Lagrangian density of conformal-affine gravity is modelled after $\mathbb{B}$, with appropriate distribution of Lie star operators so as to re-introduce the dual frame fields. The generalized conformal-affine surface topological invariant reads

$$
\mathbb{Y}=-\frac{1}{2 l^{2}}\left[\begin{array}{c}
\theta_{\mathcal{A}}\left(\mathcal{A}_{a}^{b} \wedge \widehat{R}_{b}{ }^{a}+\frac{1}{3} \mathcal{A}_{a}^{b} \wedge \mathcal{A}_{b}{ }^{c} \wedge \mathcal{A}_{c}{ }^{a}\right)+ \\
-\theta_{\mathcal{V}} \mathcal{V}_{a} \wedge \mathbf{T}^{\alpha}+\theta_{\Phi} \Phi \wedge \mathcal{Z}
\end{array}\right]
$$


where $\mathbf{T}^{\alpha}:=\mathcal{T}^{\alpha}+\mathcal{K}^{\alpha}$. The associated total conformal-affine boundary term is given by,

$$
\mathbb{B}=\frac{1}{2 l^{2}}\left[\begin{array}{c}
\widehat{R}_{\beta \alpha} \wedge \mathbf{B}^{\beta \alpha}+\Sigma^{[\beta \alpha]} \wedge \Sigma_{[\beta \alpha]}-\widehat{R}^{\alpha \beta} \wedge \widehat{R}_{\alpha \beta}-\mathcal{Z} \wedge \mathcal{Z}+ \\
+\mathcal{K}_{\alpha} \wedge \mathcal{K}^{\alpha}+\mathcal{T}_{\alpha} \wedge \mathcal{T}^{\alpha}-\Phi \wedge\left(V_{\alpha} \wedge \mathcal{T}^{\alpha}+\vartheta_{\alpha} \wedge \mathcal{K}^{\alpha}\right)+ \\
-\Upsilon_{\alpha \beta} \wedge\left(V^{\alpha} \wedge \mathcal{T}^{\beta}+\vartheta^{\alpha} \wedge \mathcal{K}^{\beta}\right)
\end{array}\right]
$$

Using the boundary term (164) as a guide, we choose $[48,51,54,56,66]$ an action of form

$$
I=\int_{\mathcal{M}}\left\{\begin{array}{c}
d\left(\mathcal{V}^{\alpha} \wedge \mathbf{T}_{\alpha}\right)+\widehat{R}^{\alpha \beta} \wedge \Sigma_{\star \alpha \beta}+\mathcal{B}_{\star \alpha \beta} \wedge \mathcal{B}^{\alpha \beta}+\Psi_{\star \alpha \beta} \wedge \Psi^{\alpha \beta}+\eta_{\star \alpha \beta} \wedge \eta^{\alpha \beta} \\
-\frac{1}{2}\left(\mathcal{R}_{\star \mu \nu} \wedge \mathcal{R}^{\mu \nu}+\mathcal{Z} \wedge \star \mathcal{Z}\right)+\mathcal{T}_{\star \alpha} \wedge \mathcal{T}^{\alpha}+\mathcal{K}_{\star \alpha} \wedge \mathcal{K}^{\alpha}+ \\
-\Phi \wedge\left(\mathcal{T}^{\star \alpha} \wedge V_{\alpha}+\mathcal{K}^{\star \alpha} \wedge \vartheta_{\alpha}\right)-\Upsilon_{\alpha \beta} \wedge\left(V^{\alpha} \wedge \mathcal{T}^{\star \beta}+\vartheta^{\alpha} \wedge \mathcal{K}^{\star \beta}\right) .
\end{array}\right\}
$$

Note that the action integral (165) is invariant under Lorentz rather than conformal-affine transformations. The Lie star $\star$ operator is defined as $\star V_{\alpha}=\frac{1}{3 !} \eta_{\alpha \beta \mu \nu} V^{\beta} \wedge V^{\mu} \wedge V^{\nu}$.

The field equations are obtained from the variation of $I$ with respect to the independant gauge potentials. It is convenient to define the functional derivatives

$$
\begin{gathered}
\frac{\delta \mathcal{L}_{\text {gauge }}}{\delta V^{\alpha}}:=-\stackrel{\mathrm{GL}}{\nabla} N_{\alpha}+\stackrel{\mathrm{V}}{\mathrm{T}}_{\alpha}, \\
\frac{\delta \mathcal{L}_{\text {gauge }}}{\delta \vartheta^{\alpha}}:=-\stackrel{\mathrm{GL}}{\nabla} M_{\alpha}+\stackrel{\vartheta}{\mathfrak{T}}_{\alpha}, \\
\mathfrak{Z}_{\alpha}^{\beta}:=\frac{\delta \mathcal{L}_{\text {gauge }}}{\delta \widehat{\Gamma}^{\alpha}}=-{ }^{\mathrm{GL}} \widehat{M}^{\beta} \widehat{M}_{\alpha}^{\beta}+\widehat{E}_{\alpha}^{\beta} .
\end{gathered}
$$

where

$$
\widehat{M}_{\beta}^{\alpha}:=-\frac{\partial \mathcal{L}_{\text {gauge }}}{\partial \widehat{R}_{\alpha}^{\beta}}, \widehat{E}_{\alpha}^{\beta}:=\frac{\partial \mathcal{L}_{\text {gauge }}}{\partial \widehat{\Gamma}_{\beta}^{\alpha}}, \stackrel{\mathrm{T}}{\alpha}_{\alpha}:=\frac{\partial \mathcal{L}_{\text {gauge }}}{\partial V^{\alpha}}, \stackrel{\vartheta}{\mathfrak{T}}_{\alpha}:=\frac{\partial \mathcal{L}_{\text {gauge }}}{\partial \vartheta^{\alpha}}, \Theta:=\frac{\partial \mathcal{L}_{\text {gauge }}}{\partial \Phi} .
$$

The gauge field momenta are defined by

$$
\begin{gathered}
N_{\alpha}:=-\frac{\partial \mathcal{L}_{\text {gauge }}}{\partial \mathcal{T}^{\alpha}}, M_{\alpha}:=-\frac{\partial \mathcal{L}_{\text {gauge }}}{\partial \mathcal{K}^{\alpha}}, \Xi:=-\frac{\partial \mathcal{L}_{\text {gauge }}}{\partial \mathcal{Z}} \\
\widehat{M}_{[\alpha \beta]}:=N_{\alpha \beta}=-o_{[\alpha \mid \gamma} \frac{\partial \mathcal{L}_{\text {gauge }}}{\partial \mathcal{R}_{\gamma}^{\mid \beta]}}, \widehat{M}_{(\alpha \beta)}:=M_{\alpha \beta}=-2 o_{(\alpha \mid \gamma} \frac{\partial \mathcal{L}_{\text {gauge }}}{\left.\partial \mathfrak{R}_{\gamma} \beta\right)} .
\end{gathered}
$$

Furthermore, the shear (gauge field deformation) and hypermomentum current forms are given by

$$
\widehat{E}_{(\alpha \beta)}:=U_{\alpha \beta}=-V_{(\alpha} \wedge\left(M_{\beta)}+N_{\beta)}\right)-M_{\alpha \beta}, \widehat{E}_{[\alpha \beta]}:=E_{\alpha \beta}=-V_{[\alpha} \wedge\left(M_{\beta]}+N_{\beta]}\right),
$$

The analogue of the Einstein equations read

$$
G_{\alpha}+\Lambda \widehat{\eta}_{\alpha}+\stackrel{\mathrm{GL}}{\nabla} \mathcal{T}_{\star \alpha}+\stackrel{\mathrm{V}}{\mathfrak{T}_{\alpha}}=0
$$

with Einstein-like three-form

$$
G_{\alpha}=\left(\mathcal{R}^{\beta \gamma}+\Upsilon_{\rho}^{[\beta \mid} \wedge \Upsilon^{\mid \gamma] \rho}\right) \wedge\left(\eta_{\beta \gamma \alpha}+\star\left[B_{\beta \gamma} \wedge \vartheta_{\alpha}\right]\right)
$$

coupling (cosmological) constant $\Lambda$ and mixed three-form $\widehat{\eta}_{\alpha}=\eta_{\alpha}+\star\left(\vartheta_{\alpha} \wedge V_{\beta}\right) \wedge V^{\beta}$. Let us observe that $G_{\alpha}$ includes symmetric $G L_{4}(\Upsilon)$ as well as special conformal $(\vartheta)$ contributions. The gauge field 3 -form $\mathfrak{T}_{\alpha}$ is given by

$$
\begin{aligned}
\stackrel{\mathrm{V}}{\alpha}_{\alpha}= & \left\langle\mathcal{L}_{\text {gauge }} \mid e_{\alpha}\right\rangle+\left\langle\mathcal{Z} \mid e_{\alpha}\right\rangle \wedge \Xi+\left\langle\mathcal{T}^{\beta} \mid e_{\alpha}\right\rangle \wedge N_{\beta}+ \\
& +\left\langle\mathcal{K}^{\beta} \mid e_{\alpha}\right\rangle \wedge M_{\beta}+\left\langle\mathcal{R}_{\gamma}^{\beta} \mid e_{\alpha}\right\rangle \wedge N_{\beta}^{\gamma}+\frac{1}{2}\left\langle\Re_{\gamma}^{\beta} \mid e_{\alpha}\right\rangle M_{\beta}^{\gamma},
\end{aligned}
$$


We remark that to interpret Eqs.1171) as the gravitational field equation analogous to the Einstein equations, we must transform from the Lie algebra index $\alpha$ to the spacetime basis index $k$ by contracting over the former $(\alpha)$ with the conformal-affine tetrads $e_{k}^{\alpha}$. This fact is relevant to read gravity in holonomic and anholonomic frames respectively. It is

$$
\begin{aligned}
\stackrel{\mathrm{V}}{\mathrm{V}}_{\alpha}= & \mathfrak{T}_{\alpha}[\mathcal{T}]+\mathfrak{T}_{\alpha}[\mathcal{K}]+\mathfrak{T}_{\alpha}[\mathcal{R}]+\mathfrak{T}_{\alpha}[Z]-\left\langle\mathcal{T}^{\beta} \mid e_{\alpha}\right\rangle \wedge N_{\beta}-\left\langle\mathcal{K}^{\beta} \mid e_{\alpha}\right\rangle \wedge M_{\beta}+ \\
& -\left\langle\mathcal{R}_{\gamma}^{\beta} \mid e_{\alpha}\right\rangle \wedge N_{\beta}^{\gamma}-\left\langle\mathcal{Z} \mid e_{\alpha}\right\rangle \wedge \Xi+\Psi_{\star \alpha \beta} \wedge \vartheta^{\beta}+\left\langle\Sigma_{\star \gamma \beta} \mid e_{\alpha}\right\rangle \wedge \widehat{R}^{\alpha \beta}+ \\
& +\left\langle\Upsilon^{\gamma \beta} \wedge\left(V_{\gamma} \wedge \mathcal{T}_{\star \beta}+\vartheta_{\gamma} \wedge \mathcal{K}_{\star \beta}\right) \mid e_{\alpha}\right\rangle+\Sigma_{\star \gamma \beta} \wedge\left\langle\widehat{R}^{\gamma \beta} \mid e_{\alpha}\right\rangle+ \\
& \mathcal{B}_{\star \gamma \beta} \wedge\left\langle\mathcal{B}^{\gamma \beta} \mid e_{\alpha}\right\rangle+\left\langle\mathcal{B}_{\star \gamma \beta} \mid e_{\alpha}\right\rangle \wedge \mathcal{B}^{\gamma \beta}+\left\langle\Psi_{\star \gamma \beta} \mid e_{\alpha}\right\rangle \wedge \Psi^{\gamma \beta}
\end{aligned}
$$

respectively, with

$$
\begin{gathered}
\mathfrak{T}_{\alpha}[\mathcal{R}]=\frac{1}{2} a_{1}\left(\mathcal{R}_{\rho \gamma} \wedge\left\langle\mathcal{R}^{\star \rho \gamma} \mid e_{\alpha}\right\rangle-\left\langle\mathcal{R}_{\rho \gamma} \mid e_{\alpha}\right\rangle \wedge \mathcal{R}^{\star \rho \gamma}\right), \\
\mathfrak{T}_{\alpha}[\mathcal{T}]=\frac{1}{2} a_{2}\left(\mathcal{T}_{\gamma} \wedge\left\langle\mathcal{T}^{\star \gamma} \mid e_{\alpha}\right\rangle-\left\langle\mathcal{T}_{\gamma} \mid e_{\alpha}\right\rangle \wedge \mathcal{T}^{\star \gamma}\right), \\
\mathfrak{T}_{\alpha}[\mathcal{K}]=\frac{1}{2} a_{3}\left(\mathcal{K}_{\gamma} \wedge\left\langle\mathcal{K}^{\star \gamma} \mid e_{\alpha}\right\rangle-\left\langle\mathcal{K}_{\gamma} \mid e_{\alpha}\right\rangle \wedge \mathcal{K}^{\star \gamma}\right), \\
\mathfrak{T}_{\alpha}[Z]=\frac{1}{2} a_{4}\left(d \Phi \wedge\left\langle\star d \Phi \mid e_{\alpha}\right\rangle-\left\langle d \Phi \mid e_{\alpha}\right\rangle \wedge \star d \Phi\right) .
\end{gathered}
$$

From the variation of $I$ with respect to $\vartheta^{\alpha}$ we get

$$
\mathfrak{G}_{\alpha}+\Lambda \widehat{\omega}_{\alpha}+\stackrel{\dagger}{\dagger} \nabla \mathcal{K}_{\star \alpha}+\stackrel{\vartheta}{\mathfrak{T}}_{\alpha}=0
$$

where, in analogy to Eqs.(171), we have

$$
\mathfrak{G}_{\alpha}=h_{i}^{\alpha}\left(\mathcal{R}^{\beta \gamma}+\Upsilon_{\rho}^{[\beta \mid} \wedge \Upsilon^{\mid \gamma] \rho}\right) \wedge\left(\omega_{\beta \gamma \alpha}+\star\left[\mathcal{B}_{\beta \gamma} \wedge V_{\alpha}\right]\right),
$$

where $\widehat{\omega}_{\alpha}=\omega_{\alpha}+\star\left(\vartheta_{\alpha} \wedge V_{\beta}\right) \wedge \vartheta^{\beta}$. The quantity $\stackrel{\vartheta}{\mathfrak{T}}_{i}=h_{i}^{\alpha} \stackrel{\vartheta}{\mathfrak{T}_{\alpha}}$ is similar to (172) but with the algebra basis $e_{\alpha}$ replaced by $h_{\alpha}$ and the conformal-affine tetrad components $e_{i}^{\alpha}$ replaced by $h_{i}^{\alpha}$. The two gravitational field equations (171) and (176) are $P-\Delta$ symmetric. We may say that they exhibit $P-\Delta$ duality symmetry invariance.

From the variational equation for $\stackrel{\circ}{\Gamma}_{\alpha}^{\beta}$, we obtain the conformal-affine gravitational analogue of the Yang-Millstorsion type field equation,

$$
\stackrel{\circ}{\nabla} \star \mathcal{R}_{\alpha}^{\beta}+\stackrel{\circ}{\nabla} \star \Sigma_{\alpha}^{\beta}+\left(V^{\beta} \wedge \mathcal{T}_{\star \alpha}+\vartheta^{\beta} \wedge \mathcal{K}_{\star \alpha}\right)=0
$$

Variation of $I$ with respect to $\Upsilon_{\alpha}^{\beta}$ leads to

$$
\stackrel{\circ}{\nabla} \star \Sigma_{\alpha \beta}-\Upsilon_{(\alpha \mid}^{\gamma} \wedge \Sigma_{\star \gamma \mid \beta)}+V_{(\alpha} \wedge \mathcal{T}_{\star \beta)}+\vartheta_{(\alpha} \wedge \mathcal{K}_{\star \beta)}=0 .
$$

Finally, from the variational equation for $\Phi$, the gravi-scalar field equation is given by

$$
d \star d \Phi+V_{\alpha} \wedge \mathcal{T}^{\star \alpha}+\vartheta_{\alpha} \wedge \mathcal{K}^{\star \alpha}=0
$$

In conclusions, the field equations of conformal-affine gravity have been obtained in this section. The analogue of the Einstein equation, obtained from variation of $I$ with respect to the coframe $V$, is characterized by an Einstein-like 3 -form that includes symmetric $G L_{4}$ as well as special conformal contributions. Moreover, the field equation in (171) contains a non-trivial torsion contribution. Performing a $P-\Delta$ transformation ( i.e. $V \rightarrow \vartheta, \mathcal{T} \rightarrow \mathcal{K}, D \rightarrow-D$ ) on (171) we obtain (176). This result may also be obtained directly by varying $I$ with respect $\vartheta$. A mixed conformal-affine cosmological constant term arises in (171), (176)) as a consequence of the structure of the 2 -form $\mathbb{R}_{\beta}^{\alpha}$. This result can be extremely interesting from a physical viewpoint in order to envisage a mechanism capable of producing the "observed" cosmological constant (see also [14, 15]). 
The field equation (177) is a Yang-Mills-like equation that represents the generalization of the Gauss torsion-free equation $\nabla \star B^{\alpha \beta}=0$. In our case, we considered a mixed volume form involving both $V$ and $\vartheta$ leading to the substitution $B^{\alpha \beta} \rightarrow \Sigma^{\alpha \beta}$. Additionally, even in the case of vanishing $T^{\rho}=\stackrel{\circ}{\nabla} V^{\rho}$, the conformal-affine torsion depends on the dilaton potential $\Phi$ which in general is non-vanishing. A similar argument holds for the special conformal quantity $\mathcal{K}^{\rho}$. Admitting the quadratic curvature term $\mathcal{R}_{\alpha}^{\beta} \wedge \star \mathcal{R}_{\beta}^{\alpha}$ in the gauge Lagrangian it becomes clear how we draw the analogy between (177) and the Gauss equation. Equation (178) follow from similar considerations as (177), the significant differences being the lack of a ${\stackrel{\circ}{\nabla} \star \mathfrak{R}_{\alpha}^{\beta}}$ counterpart to $\stackrel{\circ}{\nabla} \star \mathcal{R}_{\alpha}^{\beta}$ since $\star \Re_{\alpha}^{\beta}=0$. Finally, (179) involves both $\mathcal{T}^{\rho}$ and $\mathcal{K}^{\rho}$ in conjunction with a term that resembles the source-free Maxwell equations with the dilaton potential playing a similar role to the electromagnetic vector potential.

\section{CONCLUSIONS AND PERSPECTIVES}

In this review paper, after a summary of the bundle approach to the gauge theories with a discussion, in particular, of the bundle structure of gravitation, a nonlinearly realized representation of the local conformal-affine group has been determined. Before the physical applications, we have reviewed, in details, all the mathematical tools to show that gravity and spin are the results of the local conformal-affine group so then it is possible to deal with an Invariance Induced Gravity. It has been found that the nonlinear Lorentz transformation law contains contributions from the linear Lorentz parameter as well as conformal and shear contributions via the nonlinear 4-boosts and symmetric $G L_{4}$ parameters. We have identified the pullback of the nonlinear translational connection coefficient to $M$ as a spacetime coframe. In this way, the frame fields of the theory are obtained from the (nonlinear) gauge prescription. The mixed index coframe component (tetrad) is used to convert from Lie algebra indices into spacetime indices. The spacetime metric is a secondary object constructed (induced!) from the constant $H$ group metric and the tetrads.

The gauge fields $\Gamma^{\alpha \beta}$ are the analogues of the Christoffel connection coefficients of General Relativity and serve as the gravitational gauge potentials used to define covariant derivative operators. The gauge fields $\vartheta$, $\Phi$, and $\Upsilon$ encode information regarding special conformal, dilatonic and deformational degrees of freedom of the bundle manifold [40]. The spacetime geometry is therefore determined by gauge field interactions as in the so called Emergent Gravity [55].

Furthermore, the bundle curvature and the Bianchi identities have been determined and then the gauge Lagrangian density have been modelled after the boundary topological invariants have been defined. As a consequence of this approach, no mixed field strength terms involving different components of the total curvature arise in the action. The analogue of the Einstein equations contains a non-trivial torsion contribution which is directly related to the spin fields of the theory (see also [56]). The Einstein-like three-form includes symmetric $G L_{4}$ as well as special conformal contributions. A mixed translational-conformal cosmological constant term arises due to the structure of the generalized curvature of the manifold. We also obtain a Yang-Mills-like equation that represents the generalization of the Gauss torsion-free equation. Variation of $I$ with respect to $\Upsilon_{\alpha}^{\beta}$ leads to a constraint equation relating the $G L_{4}$ deformation gauge field to the translational and special conformal field strengths. The gravi-scalar field equation has non-vanishing translational and special conformal contributions. As a concluding remark, we can say that gravity (and in general any gauge field) can be derived as the nonlinear realization of a local conformal-affine symmetry group and then gravity can be considered an interaction induced from invariance properties. This approach can be adopted also to generalized theories of gravity [57, 58, 59] as we are going to do in a forthcoming paper.

[1] R. Utiyama, Phys. Rev. 101 (1956) 1597.

[2] C. N. Yang and R. L. Mills, Phys. Rev. 96 (1954) 191.

[3] T.W. Kibble, J. Math. Phys. 2 (1960) 212.

[4] E. Cartan, Ann. Ec. Norm. 42 (1925) 17.

[5] D.W. Sciama, On the analog between charge and spin in General Relativity, in Recent Developments in General Relativity, Festschrift for Leopold Infeld, (1962) 415, Pergamon Press, New York.

[6] R. Finkelstein, Ann. Phys. 12, 200 (1961)

[7] F. W. Hehl and B.K. Datta, J. Math. Phys. 12 (1971) 1334.

[8] F. W. Hehl, P. von der Heyde, G. D. Kerlick, and J. M. Nester Rev. Mod. Phys. 48 (1976) 393.

[9] F. Mansouri and L.N. Chang, Phys. Rev. D13 (1976) 3192.

[10] F. Mansouri, Phys. Rev. Lett. 42 (1979) 1021.

[11] G. Grignani and G. Nardelli, Phys. Rev. D45 (1992) 2719.

[12] L. N. Chang, K. I. Macrae and F. Mansouri, Phys. Rev. D13 (1976) 235.

[13] F. W. Hehl and J. D. McCrea, Found. of Phys. 16 (1986) 267. 
[14] S. Capozziello, R. Cianci, C. Stornaiolo and S. Vignolo, Class. Quantum Grav. 24 (2007) 6417.

[15] S. Capozziello, R. Cianci, C. Stornaiolo and S. Vignolo, Int. J. Geom. Methods Mod. Phys. 5, (2008) 765.

[16] G. Magnano, M. Ferraris, M. Francaviglia, Class. Quantum Grav. 7 (1990) 557.

[17] A. Inomata and M. Trikala, Phys. Rev. D19 (1978) 1665.

[18] C. G. Callan, S. Coleman, J. Wess and B. Zumino, Phys. Rev. 117 (1969) 2247.

[19] S. Coleman, J. Wess and B. Zumino, Phys. Rev. 117, (1969) 2239.

[20] C. J. Isham, A. Salam and J. Strathdee, Ann. of Phys. 62 (1971) 98.

21] A. Salam and J. Strathdee, Phys. Rev. 184, 1750 (1969); Phys. Rev. 184 (1969) 1760.

[22] A.B. Borisov and V.I. Ogievetskii, Theor. Mat. Fiz. 21, 329 (1974)

[23] E. A. Ivanov and V. I. Ogievetskii, Gauge theories as theories of spontaneous breakdown, Preprint of the Joint Institute of Nuclear Research, E2-9822 (1976) 3-10

[24] L. N. Chang et al., Phys. Rev. D17 (1978) 3168.

[25] K. S. Stelle et al., Phys. Rev. D21 (1980) 1466.

[26] E. A. Ivanov and J. Niederle, Phys. Rev. D25 (1982) 976.

[27] E. A. Ivanov and J. Niederle, Phys. Rev. D25 (1982) 988.

[28] D. Ivanenko and G. A. Sardanashvily, Phys. Rep. 94 (1983) 1.

[29] S. Capozziello and M. De Laurentis, Int. Jou. Geom. Meth. in Mod. Phys. 6 (2009) 1.

[30] G. Basini and S. Capozziello, Gen. Rel. Grav., 35 (2003) 2217.

[31] G. Basini and S. Capozziello, Int. Jou. Mod. Phys. D 15 (2006) 583.

[32] A. Lord and P. Goswami, J. Math. Phys. 27 (1986) 3051.

[33] E. A. Lord and P. Goswami, J. Math. Phys. 29 (1987) 258.

[34] Y. Ne'eman and T. Regge, Riv. Nuovo Cimento 1 (1978) 1.

[35] Y. Ne'eman and D. Sijacki, Gravity from Symmetry Breakdown of a Gauge Affine Theory, The Center for Particle Theory, University of Texas at Austin, D6-87/40 (1987).

[36] A. Lopez-Pinto, A. Tiemblo and R. Tresguerres, Class. Quant. Grav. 12 (1995) 1503.

[37] J. Julve et. al., Class. Quantum Grav. 12 (1995) 1327.

[38] R. Tresguerres and E. W. Mielke, Phys. Rev. D 62 (2000) 044004.

[39] R. Tresguerres, Phys. Rev. D66 (2002) 064025.

[40] S. Capozziello and C. Stornaiolo, Int. Jou. Geom. Methods in Mod. Phys. 5 (2008) 185.

[41] A. Tiemblo and R. Tresguerres, Gravitational contribution to fermion masses, arXiv: gr-qc/0506034 Eur. Phys. J. C 42 (2005) 437.

[42] A. Tiemblo and R. Tresguerres, Recent Res. Devel. Phys. 5 (2004) 1255

[43] A. Schwarz, Topology for Physicists, (Springer-Verlag: Berlin Heidelberg, 1994)

[44] M. Nakahara, "Geometry, Topology and Physics", Second Edition, Graduate Student Series in Physics, Institute of Physics, Bristol (2003)

[45] A. Tiemblo and R. Tresguerres, Time evolution in dynamical spacetimes, arXiv: gr-qc/9607066

[46] G. Giachetta, J. Math. Phys. 40 (1999) 939.

[47] F. W. Hehl and W. Kopczynski, J. Math. Phys. 32 (1991) 2169.

[48] S. L. Cacciatori, M. M. Caldarelli, A. Giacomini, D. Klemm, D. S. Mansi, J. Geom. Phys. 56 (2006) 2523.

[49] O. Chandia and J. Zanelli, "Torsional Topological Invariants (and their relevance for real life)", arXiv: hep-th/9708138

[50] A. Macias et. al, J.Math. Phys. 36 (1995) 5868.

[51] R. Tresguerres, J. Math. Phys. 33 (1992) 4231.

[52] R. Tucker and C. Wang, Non-Riemannian Gravitational Interactions, arXiv: gr-qc/9608055

[53] T. Dereli, M. Önder, J. Schray, R. W. Tucker, C. Wang Class. Quant. Grav. 13 (1996) L103.

[54] R. Scipioni, Class. Quant. Grav. 16 (1999) 2471.

[55] N. Seiberg, "Emergent Spacetime", Rapporteur talk at the 23rd Solvay Conference in Physics, December, 2005, arXiv:hep-th/0601234

[56] S. Capozziello, G. Lambiase, C. Stornaiolo, Annals. Phys. (Leipzig) 10 (2001) 713.

[57] S. Nojiri and S.D. Odintsov, Int. J. Geom. Methods in Mod. Phys. 4 (2007) 115.

[58] S. Capozziello and M. Francaviglia, Gen. Rel. Grav. 40(2008) 357.

[59] T.P. Sotiriou and V. Faraoni, arXiv:0805.1726 [gr-qc], at press in Phys. Rep. 2009. 